\title{
ON BORNOLOGICAL INDUCED PSEUDONEARNESS
}

\author{
DIETER LESEBERG AND ZOHREH VAZIRY
}

\begin{abstract}
Pseudonearness is considered a common tool for studying bornology, b-topology, pseudoproximity, and last but not least, classical nearness. For any pseudonear space we construct a b-completion, which generalizes the classical completion of nearness spaces. Then, b-compactification is introduced in the context of strict bornotopological extensions.
\end{abstract}

\section{INTRODUCTION}

In this paper, we generalize the classical nearness in the realm of pseudonearness. It is shown that bornologies, b-topologies, pseudoproximities and classical nearness structures have corresponding counterparts. By constructing a b-completion for any pseudonear space, we obtain a natural generalization of Herrlich's completion of a nearness space. Then, contiguous pseudonearness comes into play by considering strict bornotopological extensions. It is shown that there exists a natural correspondence between contiguous pseudonearness and equivalence classes of strict b-compactifications. Thus, the evident result for so-called saturated contiguous pseudonearness generalizes a theorem for contiguity spaces in the sense of Ivanova and Ivanov. By applying our central theorem to proxiform pseudonearness, we obtain, in the saturated case, a comparative form of the famous theorem of Lodato.

Definition 1.1. For a set $X$, let $\mathcal{B}^{X} \subset \underline{P} X$ be a non-empty subset, where $\underline{P} X$ denotes the powerset of $X$. Then, $\mathcal{B}^{X}$ is called bornology, provided it satisfies the following conditions:

(b $b_{1} B_{1} \subset B \in \mathcal{B}^{X}$ implies $B_{1} \in \mathcal{B}^{X}$;

(b) $x \in X$ implies $\{x\} \in \mathcal{B}^{X}$;

(b) $B_{1}, B_{2} \in \mathcal{B}^{X}$ implying $B_{1} \cup B_{2} \in \mathcal{B}^{X}$.

Then, the pair $\left(X, \mathcal{B}^{X}\right)$ is called a bornological space.

Remark 1.2. Here, we point out that this definition is equivalent to that given by H. Hogbe-Nlend,[3] in 1977.

Definition 1.3. For bornologies $\mathcal{B}^{X}$ and $\mathcal{B}^{Y}$ a function $f: X \longrightarrow Y$ is called

(i) bounded, provided it satisfies the condition

$$
\text { (b) }\left\{f[B]: B \in \mathcal{B}^{X}\right\} \subset \mathcal{B}^{Y} \text {; }
$$

$M S C$ (2010): primary 54D35, 54A05, 54D10, 54D30, 54E05, 54E17.

Keywords: bounded topology, pseudonearness, nearness, proximity, b-compactness, b-completeness, topological extension, contiguous, bornology, b-topology, separated, strict extension. 
(ii) rebounded, provided it satisfies the condition (rb) $B \in \mathcal{B}^{Y}$ implies $f^{-1}[B] \in \mathcal{B}^{Y}$

(iii) bibounded, provided it is bounded and rebounded, (bib).

Remark 1.4. Now, we denote by BORN the category whose objects are bornological spaces and whose morphisms are bounded maps and, moreover, by 2BORN the category whose objects are bornological spaces and whose morphisms are bibounded maps. In this context, compare also the description in Leseberg and Vaziry [6]. In addition, we note that BORN can be regarded as a full subcategory of BOUND, the category of bound spaces and bounded maps forming a quasitopos [6].

Now, every bornology can be naturally equipped with an operator $N^{b^{X}}$ : $\mathcal{B}^{X} \longrightarrow \underline{P}(\underline{P}(\underline{P} X))$ by setting:

$$
N^{b^{X}}(\emptyset):=\{\emptyset\}
$$

and

$$
N^{b^{X}}(B):=\left\{\mathcal{S} \subset \underline{P} X: \exists x \in B \forall F \in \mathcal{S} \cap \mathcal{B}^{X}, x \in F\right\} \text { for each } B \in \mathcal{B}^{X} \backslash\{\emptyset\} .
$$

Then, the pair $\left(\mathcal{B}^{X}, N^{b^{X}}\right)$ satisfies the conditions for being a pseudonearness $\left(\mathcal{B}^{X}, N\right)$ on $X$, where, in the following, the triple $\left(X, \mathcal{B}^{X}, N\right)$ is called a pseudonear space. In fact, $\left(\mathcal{B}^{X}, N\right)$ has to fulfill the below listed conditions, i.e.

$\left(\operatorname{psn}_{1}\right) B \in \mathcal{B}^{X} \backslash\{\emptyset\}$ and $\mathcal{S} \in N(B)$ implying $\{B\} \cup \mathcal{S} \in \cap\left\{N(F): F \in\left(\mathcal{S} \cap \mathcal{B}^{X}\right) \cup\right.$ $\{B\}\}$ (symmetry);

$\left(\operatorname{psn}_{2}\right) B \in \mathcal{B}^{X}$ implies $c l_{N}(B) \in \mathcal{B}^{X}$, where in general $c l_{N}(B):=\{x \in X$ : $\{B\} \in N(\{x\})\}$ (hull-bounded);

$\left(\operatorname{psn}_{3}\right) B \in \mathcal{B}^{X}$ and $\mathcal{S} \cap \mathcal{B}^{X} \in N(B), \mathcal{S} \subset \underline{P} X$ implying $\mathcal{S} \in N(B)$ (b-absorbed);

$\left(\operatorname{psn}_{4}\right) B \in \mathcal{B}^{X}$ implies $\mathcal{B}^{X} \notin N(B) \neq \emptyset$ (fullness-conditions);

$\left.\left(\operatorname{psn}_{5}\right) \mathcal{S} \in N(\emptyset\}\right)$ implies $\mathcal{S}=\emptyset$ (zero-set condition);

$\left(\operatorname{psn}_{6}\right) B \in \mathcal{B}^{X}$ and $\mathcal{S}_{1} \ll \mathcal{S} \in N(B)$ implying $\mathcal{S}_{1} \in N(B)$ (corefinement), where $\mathcal{S}_{1} \ll \mathcal{S}$ iff $\forall F_{1} \in \mathcal{S}_{1} \exists F \in S$ such that $F_{1} \supset F$;

$\left(\operatorname{psn}_{7}\right) B \in \mathcal{B}^{X}$ and $\mathcal{S}_{1}, \mathcal{S}_{2} \notin N(B)$ implying $\mathcal{S}_{1} \vee \mathcal{S}_{2} \notin N(B)$ (finiteness), where $\mathcal{S}_{1} \vee \mathcal{S}_{2}:=\left\{F_{1} \cup F_{2}: F_{1} \in \mathcal{S}_{1}, F_{2} \in \mathcal{S}_{2}\right\}$

$\left(\mathrm{psn}_{8}\right) \quad x \in X$ implies $\{\{x\}\} \in N(\{x\})$ (single sets);

$\left(\operatorname{psn}_{9}\right)\left\{c l_{N}(F): F \in \mathcal{S}\right\} \in N(B), B \in \mathcal{B}^{X}$ and $\mathcal{S} \subset \underline{P} X$ implying $\mathcal{S} \in N(B)$ (density).

Definition 1.5. By PSN we denote the category whose objects are pseudonear spaces and whose morphisms are bibounded near maps (in short bin-maps), where a bibounded map $f: X \longrightarrow Y$ between pseudonear spaces $\left(X, \mathcal{B}^{X}, N\right),\left(Y, \mathcal{B}^{Y}, M\right)$ is called a bin-map, provided it also fulfills the following condition:

(n) $B \in \mathcal{B}^{X} \backslash\{\emptyset\}$ and $\mathcal{S} \in N(B)$ implying $\{f[F]: F \in \mathcal{S}\}=: f \mathcal{S} \in M(f[B])$.

Theorem 1.6. The category $\mathbf{2 B O R N}$ is isomorphic to a full subcategory of PSN.

Proof. It may easily be seen that for bornological spaces $\left(X, \mathcal{B}^{X}\right),\left(Y, \mathcal{B}^{Y}\right)$ and a function $f: X \longrightarrow Y$ the following statements are equivalent:

(i) $f:\left(X, \mathcal{B}^{X}\right) \longrightarrow\left(Y, \mathcal{B}^{Y}\right)$ is a bibounded map (in short a bib-map); 
(ii) $f:\left(X, \mathcal{B}^{X}, N^{b^{X}}\right) \longrightarrow\left(Y, \mathcal{B}^{Y}, N^{b^{Y}}\right)$ is a bin-map.

Let us call a pseudonear space $\left(X, \mathcal{B}^{X}, M\right)$ bornoform, provided it satisfies

(bf) $B \in \mathcal{B}^{X} \backslash\{\emptyset\}$ and $\mathcal{S} \in M(B)$ implying $B \cap\left(\cap\left(\mathcal{S} \cap \mathcal{B}^{X}\right)\right) \neq \emptyset$.

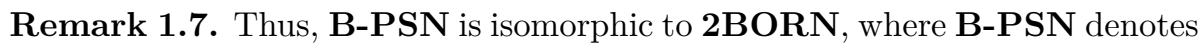
the full subcategory of PSN, whose objects are bornoform.

Nearness structures in the sense of Herrlich [2] are an important tool for a common study of topological structures and uniformities. Moreover, they are providing useful tools for the study of various kinds of strict extensions [1]. Now, in the following, we will see that they can also be regarded as special cases of pseudonear structures. In fact, let a nearness space $(X, \xi)$ be given, then we consider the pair $\left(\underline{P} X, N_{\xi}\right)$, where the operator $N_{\xi}$ is defined by setting:

$$
N_{\xi}(\emptyset):=\{\emptyset\}
$$

and

$$
N_{\xi}(B):=\{\mathcal{S} \subset \underline{P} X:\{B\} \cup \mathcal{S} \in \xi\} \text { for every } B \in \mathcal{B}^{X} \backslash\{\emptyset\} .
$$

Lemma 1.8. The pair $\left(\underline{P} X, N_{\xi}\right)$ defines a pseudonearness, and the triple

$$
\left(X, \underline{P} X, N_{\xi}\right)
$$

can therefore be regarded as a pseudonear space.

Proof. By straight-forward execution.

Definition 1.9. A pseudonear structure $\left(\mathcal{B}^{X}, M\right)$ and the corresponding space $\left(X, \mathcal{B}^{X}, M\right)$ are called saturated, provided that $X \in \mathcal{B}^{X}$ holds, and thus $\mathcal{B}^{X}=\underline{P} X$.

Lemma 1.10. For a saturated pseudonear space $\left(X, \mathcal{B}^{X}, M\right),\left(X, \eta_{M}\right)$ is a nearness space, where

$$
\eta_{M}:=\{\mathcal{A} \subset \underline{P} X: \mathcal{A} \in \cap\{M(A): A \in \mathcal{A}\}\},
$$

and the following equations are valid, i.e.

(i) $\eta_{N_{\xi}}=\eta$;

(ii) $N_{\eta_{M}}=M$.

Lemma 1.11. For nearness spaces $(X, \xi),(Y, \eta)$ and a function $f: X \longrightarrow Y$ the following statements are equivalent:

(i) $f:(X, \xi) \longrightarrow(Y, \eta)$ is a nearness map;

(ii) $f:\left(X, \underline{P} X, N_{\xi}\right) \longrightarrow\left(Y, \underline{P} Y, N_{\eta}\right)$ is a bin-map.

Notation 1.12. By SAT-PSN we denote the full subcategory of PSN, whose objects are saturated pseudonear spaces.

Theorem 1.13. The category NEAR of nearness spaces and nearness preserving maps is isomorphic to the category SAT-PSN.

Proof. By making use of the lemmas 1.8, 1.10, and 1.11, respectively. 
More generally, we note that for a pseudonearness $\left(\mathcal{B}^{X}, M\right)$ the underlying set of collections $\eta_{M}:=\left\{\mathcal{A} \subset \underline{P} X: \mathcal{A} \in \cap\left\{M(A): A \in \mathcal{A} \cap \mathcal{B}^{X}\right\}\right.$ defines a nearness on $X$. Now, we will only verify the following two conditions, i.e.

1. For $\mathcal{A}_{1}, \mathcal{A}_{2} \notin \eta_{M}$, we have $\mathcal{A}_{1} \vee \mathcal{A}_{2} \notin \eta_{M}$.

By the hypothesis, there exists $A_{1} \in \mathcal{A}_{1} \cap \mathcal{B}^{X}$ with $\mathcal{A}_{1} \notin M\left(A_{1}\right)$ and $A_{2} \in$ $\mathcal{A}_{2} \cap \mathcal{B}^{X}$ with $\mathcal{A}_{2} \notin M\left(A_{2}\right)$. Hence, $A_{1} \cup A_{2}=: A \in\left(\mathcal{A}_{1} \vee \mathcal{A}_{2}\right) \cap \mathcal{B}^{X}$ since $\mathcal{B}^{X}$ is a bornology. If $\mathcal{A}_{1} \vee \mathcal{A}_{2} \in M(A)$, then, by the finiteness, $\mathcal{A}_{1} \in M(A)$ or $\mathcal{A}_{2} \in M(A)$. By the symmetry, we get $\{A\} \cup \mathcal{A}_{1} \in M\left(A_{1}\right)$ or $\{A\} \cup \mathcal{A}_{2} \in M\left(A_{2}\right)$ and $\mathcal{A}_{1} \in M\left(A_{1}\right)$ or $\mathcal{A}_{2} \in M\left(A_{2}\right)$ leads to a contradiction.

2. Let $\mathcal{A} \subset \underline{P} X$ and $\mathcal{A} \notin \eta_{M}$. Hence, we can find $A \in \mathcal{A} \cap \mathcal{B}^{X}$ with $\mathcal{A} \notin M(A)$. By the density, we get $\left\{c l_{M}(F): F \in \mathcal{A}\right\} \notin M(A)$. But if $\left\{c l_{\eta_{M}}(F): F \in \mathcal{A}\right\} \in$ $M(A)$, we have $c l_{\eta_{M}}(F) \subset c l_{M}(F)$ for each $F \in \mathcal{A}$, because $x \in c l_{\eta_{M}}(F)$ implies $\{\{x\}, F\} \in \eta_{M}$. Hence, $\{\{x\}, F\} \in M(\{x\})$ implies $\{F\} \in M(\{x\})$ and the claim follows. But $\left\{c l_{M}(F): F \in \mathcal{A}\right\} \in M(A)$ contradicts.

In the context of nearness spaces, topological spaces come into play when one is considering topological extensions. Here, symmetric topological spaces, $T_{1}$-spaces and Hausdorff spaces are of some importance.

Now they also have counterparts in the realm of pseudonearness but here in a more generalized sense.

First, let us consider the below defined operator $t^{x}: \mathcal{B}^{X} \longrightarrow \underline{P} X$, where $x \in X$, and $\mathcal{B}^{X}$ is a bornology. We set

$$
t^{x}(\emptyset):=\emptyset
$$

and

$$
t^{x}(B):=\{x\} \cup B \text { for each } B \in \mathcal{B}^{X} \backslash\{\emptyset\} .
$$

Then, the pair $\left(\mathcal{B}^{X}, t^{x}\right)$ satisfies the conditions for being a b-topology $\left(\mathcal{B}^{X}, t\right)$ on $X$, where, in the following, the triple $\left(X, \mathcal{B}^{X}, t\right)$ is called a b-topological space. In fact, $\left(\mathcal{B}^{X}, t\right)$ has to fulfill the below listed conditions, i.e.

$\left(\mathrm{bt}_{1}\right) t(\emptyset)=\emptyset$;

$\left(\right.$ bt $\left._{2}\right) B \in \mathcal{B}^{X}$ implies $t(B) \in \mathcal{B}^{X}$;

(bt bt $_{3} \subset B \in \mathcal{B}^{X}$ implies $t\left(B_{1}\right) \subset t(B)$;

(bt bt $_{4} x \in X$ implies $\{x\} \in t(\{x\})$;

(bt b $_{5} B_{1}, B_{2} \in \mathcal{B}^{X}$ implies $t\left(B_{1} \cup B_{2}\right) \subset t\left(B_{1}\right) \cup t\left(B_{2}\right)$;

(bt 6$) B \in \mathcal{B}^{X}$ implies $t(t(B)) \subset t(B)$.

Definition 1.14. By b-TOP we denote the category whose objects are btopological spaces and whose morphisms are bibounded continuous maps (in short bic-maps), where a bibounded map $f: X \longrightarrow Y$ between b-topological spaces $\left(X, \mathcal{B}^{X}, t^{X}\right),\left(Y, \mathcal{B}^{Y}, t^{Y}\right)$ is called bic-map, provided it also fulfills the following condition:

(c) $B \in \mathcal{B}^{X} \backslash\{\emptyset\}$ implies $f\left[t^{X}(B)\right] \subset t^{Y}(f[B])$.

By b-TOP, we denote the category whose objects are b-topological spaces and whose morphisms are bic-maps.

By SATb-TOP we denote the full subcategory of b-TOP whose objects are saturated b-topological spaces. Here, a b-topological space $\left(X, \mathcal{B}^{X}, t\right)$ is called saturated if $X \in \mathcal{B}^{X}$ holds. 
Theorem 1.15. The category TOP of topological spaces and continuous maps is isomorphic to $\mathbf{S A T b}-\mathbf{T O P}$.

Proof. For a Kuratowski closure space [5] $(X, c l)$ we consider the space

$$
(X, \underline{P} X, c l)
$$

and conversely for a saturated b-topological space $\left(X, \mathcal{B}^{X}, t\right)$ the pair $(X, t)$. These assignments give rise to a functorial relationship between TOP and SATb-TOP and they establish the proposed isomorphism.

As already announced, here are the following useful definitions:

Definition 1.16. We call a b-topology $\left(\mathcal{B}^{X}, t\right)$ and the space $\left(X, \mathcal{B}^{X}, t\right)$

(i) symmetric, provided they satisfy the following condition

(s) $x, z \in X$ and $x \in t(\{z\})$ implying $z \in t(\{x\})$;

(ii) $T_{1}$, provided they satisfy the following condition

$\left(\mathrm{T}_{1}\right) x, z \in X$ and $x \in t(\{z\})$ implying $x=z$.

We denote by sb-TOP the full subcategory of b-TOP, whose objects are symmetric.

Remark 1.17. In this context, we point out that for a pseudonear space $\left(X, \mathcal{B}^{X}, N\right)$ the underlying b-topology $\left(\mathcal{B}^{X}, c l_{N^{b}}\right)$ is symmetric, meaning that the corresponding closure operator is the restriction of $c l_{N}$ onto $\mathcal{B}^{X}$.

Theorem 1.18. The category sb-TOP is isomorphic to a full subcategory of PSN.

Proof. For a symmetric b-topological space $\left(X, \mathcal{B}^{X}, t\right)$ we consider the pair $\left(\mathcal{B}^{X}, N_{t}\right)$, where $N_{t}$ is defined by setting

$$
N_{t}(\emptyset):=\{\emptyset\}
$$

and

$N_{t}(B):=\left\{\mathcal{S} \subset \underline{P} X: \cap\left\{t(F): F \in\left(\mathcal{S} \cap \mathcal{B}^{X}\right) \cup\{B\}\right\} \neq \emptyset\right\}$ for every $B \in \mathcal{B}^{X} \backslash\{\emptyset\}$.

Then, $\left(\mathcal{B}^{X}, N_{t}\right)$ forms a pseudonearness, which, in addition, is topoform.

In this context, a pseudonearness $\left(\mathcal{B}^{X}, M\right)$ and the corresponding space

$$
\left(X, \mathcal{B}^{X}, M\right)
$$

are said to be topoform, provided they satisfy the following condition:

(t) $B \in \mathcal{B}^{X} \backslash\{\emptyset\}$ and $\mathcal{S} \in M(B)$ implying $\cap\left\{c l_{M}(F): F \in\left(\mathcal{S} \cap \mathcal{B}^{X}\right) \cup\{B\}\right\} \neq$ $\emptyset$.

By T-PSN we denote the corresponding full subcategory of PSN. Moreover, the below listed equations are valid, i.e.

(i) $c l_{N_{t}}=t$;

(ii) $N_{c l_{M}}=M$, provided $\left(\mathcal{B}^{X}, M\right)$ is topoform.

And for a map $f: X \longrightarrow Y$ between b-topological spaces $\left(X, \mathcal{B}^{X}, t^{X}\right),\left(Y, \mathcal{B}^{Y}, t^{Y}\right)$ the following statements are equivalent:

(iii) $f:\left(X, \mathcal{B}^{X}, t^{X}\right) \longrightarrow\left(Y, \mathcal{B}^{Y}, t^{Y}\right)$ is a bic-map;

(iv) $f:\left(X, \mathcal{B}^{X}, N_{t^{X}}\right) \longrightarrow\left(Y, \mathcal{B}^{Y}, N_{t^{Y}}\right)$ is a bin-map. 
These facts now establish the proposed isomorphism between sb-TOP and TPSN.

As a corollary, we also note that in the saturated case, meaning that $X \in \mathcal{B}^{X}$ is valid, we obtain the embedding of $\mathbf{T O P}^{\mathbf{S}}$, the full subcategory of $\mathbf{T O P}$, whose objects are symmetric topological spaces into NEAR [2].

On the other hand, every symmetric b-topology $\left(\mathcal{B}^{X}, t\right)$ induces a compatible nearness relation between bounded sets $B_{1}, B_{2} \in \mathcal{B}^{X}$ by setting:

$$
B_{1} \delta_{t} B_{2} \quad \text { iff } \quad t\left(B_{1}\right) \cap t\left(B_{2}\right) \neq \emptyset .
$$

And if defining $c l_{\delta_{t}}(B):=\left\{x \in X:\{x\} \delta_{t} B\right\}$, then $c l_{\delta_{t}}=t$ follows, thus $\delta_{t}$ is compatible. Further, we note that by the above hypothesis, $\left(\mathcal{B}^{X}, \delta_{t}\right)$ fulfills the conditions for being a pseudoproximity $\left(\mathcal{B}^{X}, \gamma\right)$ as follows:

Definition 1.19. A pseudoproximity consists of a pair $\left(\mathcal{B}^{X}, \gamma\right)$, where $\mathcal{B}^{X}$ is a bornology and $\gamma \subset \mathcal{B}^{X} \times \mathcal{B}^{X}$ such that the following conditions are satisfied:

$\left(\operatorname{psp}_{1}\right) B \in \mathcal{B}^{X}$ implies $c l_{\gamma}(B) \in \mathcal{B}^{X}$, where $c l_{\gamma}(B):=\{x \in X:\{x\} \gamma B\}$;

$\left(\mathrm{psp}_{2}\right) B_{1} \gamma B_{2}$ implies $B_{2} \gamma B_{1}$;

( $\left.\operatorname{psp}_{3}\right) B \in \mathcal{B}^{X}$ implies $B \bar{\gamma} \emptyset$, which means $B$ is not in relation to $\emptyset$;

$\left(\operatorname{psp}_{4}\right) B_{1}, B_{2} \in \mathcal{B}^{X}$ and $B \gamma\left(B_{1} \cup B_{2}\right)$ implies $B \gamma B_{1}$ or $B \gamma B_{2}$;

$\left(\operatorname{psp}_{5}\right) B_{1} \subset B_{2}$ and $B_{1} \gamma B, B_{2} \in \mathcal{B}^{X}$ implies $B_{2} \gamma B$;

$\left(\operatorname{psp}_{6}\right) x \in X$ implies $\{x\} \gamma\{x\}$;

$\left(\operatorname{psp}_{7}\right) D \gamma c l_{\gamma}(B), B \in \mathcal{B}^{X}$ implies $D \gamma B$.

Then, the triple $\left(X, \mathcal{B}^{X}, \gamma\right)$ is called a pseudoproximity space.

A pseudoproximity space $\left(X, \mathcal{B}^{X}, \gamma\right)$ is called separated, provided it satisfies the following condition:

(sep) $x, z \in X$ and $\{x\} \gamma\{z\}$ implying $x=z$.

For pseudoproximity spaces $\left(X, \mathcal{B}^{X}, \gamma_{X}\right),\left(Y, \mathcal{B}^{Y}, \gamma_{Y}\right)$ a function $f: X \longrightarrow Y$ is called a bibounded proximal map (in short a bip-map), provided it is bibounded and satisfies

(p) $B_{1} \gamma_{X} B_{2}$ implies $f\left[B_{1}\right] \gamma_{Y} f\left[B_{2}\right]$.

Remark 1.20. Here, we point out that, in the case of saturation, meaning that $X \in \mathcal{B}^{X}$ holds, separated pseudoproximity spaces and LODATO proximity spaces are essentially the same (up to bijection). Moreover, if we denote by PSPROX the category of pseudoproximity spaces and bip-maps and by SAT-PSPROX its full subcategory of saturated objects, respectively, then we get an isomorphism between SAT-PSPROX and LOPROX the category of LODATO proximity spaces and proximal maps [7].

Now, we will embed PSPROX into PSN. Let us start with an arbitrary pseudoproximity space $\left(X, \mathcal{B}^{X}, \delta\right)$.

Proposition 1.21. For a pseudoproximity space $\left(X, \mathcal{B}^{X}, \delta\right)$, we consider the pair $\left(\mathcal{B}^{X}, N_{\delta}\right)$, where $N_{\delta}: \mathcal{B}^{X} \longrightarrow \underline{P}(\underline{P}(\underline{P} X))$ is defined by setting:

$$
N_{\delta}(\emptyset):=\{\emptyset\}
$$


and for every $B \in \mathcal{B}^{X} \backslash\{\emptyset\}$, we define

$$
\begin{aligned}
N_{\delta}(B):= & \left\{\mathcal{S} \subset \underline{P} X: \forall \mathcal{E} \subset\{B\} \cup\left(\mathcal{S} \cap \mathcal{B}^{X}\right)\right. \\
& \text { finite } \left.\left\{c l_{\delta}(E): E \in \mathcal{E}\right\} \ll\{A, D\} \text { for some } A, D \in \mathcal{B}^{X} \text { with } A \delta D\right\} .
\end{aligned}
$$

Then, $\left(\mathcal{B}^{X}, N_{\delta}\right)$ is a pseudonear structure (pseudonearness).

Moreover, if we put for such a pseudonearness $\left(\mathcal{B}^{X}, M\right)$,

$$
B_{1} \gamma_{M} B_{2} \quad \text { iff } \quad\left\{B_{1}, B_{2}\right\} \in M\left(B_{1}\right) \cap M\left(B_{2}\right),
$$

then the following statements are equivalent:

$$
B_{1} \gamma_{N_{\delta}} B_{2} \quad \text { iff } \quad B_{1} \delta B_{2} \quad \text { (compatibility). }
$$

Proof. First, we will show that the latter equivalence is valid. So, let $B_{1} \gamma_{N_{\delta}} B_{2}$.

We put $\mathcal{E}:=\left\{B_{1}, B_{2}\right\}$, then $\mathcal{E} \subset\left\{B_{1}\right\} \cup\left\{B_{1}, B_{2}\right\}$ and/or $\mathcal{E} \subset\left\{B_{2}\right\} \cup\left\{B_{1}, B_{2}\right\}$ are finite. By the hypothesis, we can choose $A, D \in \mathcal{B}^{X}$ with $\left\{c l_{\delta}\left(B_{1}\right), c l_{\delta}\left(B_{2}\right)\right\} \ll$ $\{A, D\}$ with $A \delta D$.

In the cases that $c l_{\delta}\left(B_{1}\right) \supset A$ or $c l_{\delta}\left(B_{1}\right) \supset D$ and analogously if $c l_{\delta}\left(B_{2}\right) \supset A$ or $c l_{\delta}\left(B_{2}\right) \supset D$, we obtain $c l_{\delta}\left(B_{1}\right) \delta c l_{\delta}\left(B_{2}\right)$. Hence, $B_{1} \delta B_{2}$ follows.

Conversely, let $B_{1} \delta B_{2}$. Hence, $\left\{B_{1}, B_{2}\right\} \in N_{\delta}\left(B_{1}\right) \cap N_{\delta}\left(B_{2}\right)$ because for $\mathcal{E} \subset$ $\left\{B_{1}\right\} \cup\left\{B_{1}, B_{2}\right\}$ finite and without restriction $\mathcal{E} \neq \emptyset$, we have $\left\{c l_{\delta}(E): E \in \mathcal{E}\right\} \ll$ $\left\{B_{1}, B_{2}\right\}$. Analogously, this also holds if $\mathcal{E} \subset\left\{B_{2}\right\} \cup\left\{B_{1}, B_{2}\right\}$ is finite. And consequently, $B_{1} \gamma_{N_{\delta}} B_{2}$ follows.

$\left(\mathcal{B}^{X}, N_{\delta}\right)$ is symmetric. So, let for $B \in \mathcal{B}^{X} \backslash\{\emptyset\}, \mathcal{S} \in N_{\delta}(B)$. We have to show

(1) $\{B\} \cup \mathcal{S} \in N_{\delta}(B)$ and

(2) $\{B\} \cup \mathcal{S} \in \cap\left\{N_{\delta}(F): F \in \mathcal{S} \cap \mathcal{B}^{X}\right\}$.

To (1): Let $\mathcal{E} \subset\{B\} \cup\left((\{B\} \cup \mathcal{S}) \cap \mathcal{B}^{X}\right)$ be finite. Hence, $\mathcal{E} \subset\{B\} \cup\left(\mathcal{S} \cap \mathcal{B}^{X}\right)$ is valid and, by the hypothesis, we have $\left\{c l_{\delta}(E): E \in \mathcal{E}\right\} \ll\{A, D\}$ for some $A, D \in \mathcal{B}^{X}$ with $A \delta D$. Thus, $\{B\} \cup \mathcal{S} \in N_{\delta}(B)$.

To (2): Now, let $F \in \mathcal{S} \cap \mathcal{B}^{X}$ and $\mathcal{E} \subset\{F\} \cup\left((\{B\} \cup \mathcal{S}) \cap \mathcal{B}^{X}\right)$ be finite. Hence, $\mathcal{E} \subset\{B\} \cup\left(\mathcal{S} \cap \mathcal{B}^{X}\right)$ follows and, by applying the hypothesis, $\{B\} \cup \mathcal{S} \in N_{\delta}(F)$ results.

$\left(\mathcal{B}^{X}, N_{\delta}\right)$ is hull-bounded. Let $B \in \mathcal{B}^{X} \backslash\{\emptyset\}$ and $x \in c_{N_{\delta}}(B)$. Hence, by the definition, $\{B\} \in N_{\delta}(\{x\})$ is valid. But $N_{\delta}$ is symmetric so that $\{\{x\}\} \cup\{B\} \in$ $N_{\delta}(\{x\})$ can be deduced and finally, $\{\{x\}\} \cup\{B\} \in N_{\delta}(B)$ results. Both of them show $\{\{x\}, B\} \in N_{\delta}(\{x\}) \cap N_{\delta}(B)$ and consequently, $\{x\} \gamma_{N_{\delta}} B$ is valid. By applying the already proved equivalence, we obtain $\{x\} \delta B$, which implies $x \in$ $c l_{\delta}(B)$. But $c l_{\delta}(B) \in \mathcal{B}^{X}$, by applying ( $\left.\operatorname{psp}_{1}\right)$, and thus the claim is proved by $\left(b_{1}\right)$.

Evidently, $\left(\mathcal{B}^{X}, N_{\delta}\right)$ is b-absorbed and fulfills the fullness-conditions or zero-set condition.

Next, let without restriction $\mathcal{S}_{1} \ll \mathcal{S} \in N_{\delta}(B), B \in \mathcal{B}^{X} \backslash\{\emptyset\}$. Further, let $\mathcal{E}_{1} \subset\{B\} \cup\left(\mathcal{S}_{1} \cap \mathcal{B}^{X}\right)$ be finite. For each $E_{1} \in \mathcal{E}_{1}$, choose $F_{E_{1}} \in\{B\} \cup\left(\mathcal{S} \cap \mathcal{B}^{X}\right)$ with $E_{1} \supset F_{E_{1}}$ (note that $\mathcal{B}^{X}$ is closed under the formation of subsets).

We put $\mathcal{E}:=\left\{F_{E_{1}}: E_{1} \in \mathcal{E}_{1}\right\}$, then $\mathcal{E} \subset\{B\} \cup\left(\mathcal{S} \cap \mathcal{B}^{X}\right\}$ is finite, and by the hypothesis, we get $\left\{c l_{\delta}\left(F_{E_{1}}\right): E_{1} \in \mathcal{E}_{1}\right\} \ll\{A, D\}$ with $A \delta D$. But for $E_{1} \in \mathcal{E}_{1}$, we have $E_{1} \supset F_{E_{1}}$. Hence, $c l_{\delta}\left(E_{1}\right) \supset c l_{\delta}\left(F_{E_{1}}\right)$, which shows $\mathcal{S}_{1} \in N_{\delta}(B)$. Thus, $\left(\mathcal{B}^{X}, N_{\delta}\right)$ satisfies the corefinement condition $\left(\operatorname{psn}_{6}\right)$. 
Concerning the finiteness, let without restriction $\mathcal{S}_{1}, \mathcal{S}_{2} \notin N_{\delta}(B), B \in \mathcal{B}^{X} \backslash\{\emptyset\}$. By the definition, we can find $\mathcal{E}_{1} \subset\{B\} \cup\left(\mathcal{S}_{1} \cap \mathcal{B}^{X}\right)$ finite and $\mathcal{E}_{2} \subset\{B\} \cup\left(\mathcal{S}_{2} \cap \mathcal{B}^{X}\right)$ finite such that for each $A, D \in \mathcal{B}^{X}$ with $A \delta D,\left\{c l_{\delta}\left(E_{1}\right): E_{1} \in \mathcal{E}_{1}\right\}<\nless\{A, D\}$ or $\left\{c l_{\delta}\left(E_{2}\right): E_{2} \in \mathcal{E}_{2}\right\}<\nless\{A, D\}$. So we can find $E_{1} \in \mathcal{E}_{1}, E_{2} \in \mathcal{E}_{2}$ with $c l_{\delta}\left(E_{1}\right) \nsupseteq A, D, c l_{\delta}\left(E_{2}\right) \nsupseteq A, D$ for each $A, D$ with $A \delta D$.

Consequently, $E_{1} \neq B \neq E_{2}$ for these chosen elements. Hence, $E_{1} \in \mathcal{S}_{1} \cap \mathcal{B}^{X}$ and $E_{2} \in \mathcal{S}_{2} \cap \mathcal{B}^{X}$ follows. By setting $\mathcal{E}:=\left\{E_{1} \cup E_{2}\right\}$, we obtain $\mathcal{E} \subset\{B\} \cup\left(\mathcal{S}_{1} \vee\right.$ $\left.\mathcal{S}_{2}\right) \cap \mathcal{B}^{X}$ is finite because $\mathcal{B}^{X}$ is a bornology. If $c_{\delta}\left(E_{1} \cup E_{2}\right) \ll\{A, D\}$ with $A \delta D$, then without restriction, let $c l_{\delta}\left(E_{1}\right) \cup c l_{\delta}\left(E_{2}\right)$ be a superset of $A$. Consequently, $c l_{\delta}\left(E_{1}\right) \delta D$ or $c l_{\delta}\left(E_{2}\right) \delta D$ are valid implying $c l_{\delta}\left(E_{1}\right) \nsupseteq E_{1}$ or $c l_{\delta}\left(E_{2}\right) \nsupseteq E_{2}$ by the supposition, which contradicts. Evidently, $\left(\mathcal{B}^{X}, N_{\delta}\right)$ satisfies the single sets condition.

Now, finally, we will show the density of $\left(\mathcal{B}^{X}, N_{\delta}\right)$. To this aim, let without restriction $\mathcal{S} \notin N_{\delta}(B), B \in \mathcal{B}^{X} \backslash\{\emptyset\}$. Our goal is to verify $\left\{c l_{N_{\delta}}(F): F \in \mathcal{S}\right\} \notin$ $N_{\delta}(B)$. By the hypothesis, we can find $\mathcal{E} \subset\{B\} \cup\left(\mathcal{S} \cap \mathcal{B}^{X}\right)$ finite such that $\left\{c l_{\delta}(E): E \in \mathcal{E}\right\}<\nless\{A, D\}$ with $A \delta D$ for every $A, D \in \mathcal{B}^{X}$. Choose $E \in \mathcal{E}$ with $E \in \mathcal{S} \cap \mathcal{B}^{X}$ and put $\overline{\mathcal{E}}:=\left\{c l_{N_{\delta}}(E)\right\}$. If $\left\{c l_{N_{\delta}}(F): F \in \mathcal{S}\right\} \in N_{\delta}(B)$ and since $\overline{\mathcal{E}} \subset\{B\} \cup\left(\left\{c l_{N_{\delta}}(F): F \in \mathcal{S}\right\} \cap \mathcal{B}^{X}\right)=\{B\} \cup\left\{c l_{N_{\delta}}(F): F \in \mathcal{S} \cap \mathcal{B}^{X}\right\}$ is finite, we get $\left\{c l_{\delta}\left(c l_{N_{\delta}}(E)\right)\right\} \ll\{A, D\}$ with $A \delta D$. Thus, $c l_{\delta}(E) \supset A$ or $c l_{\delta}(E) \supset D$. Without restriction, let $c l_{\delta}(E) \supset A$. Since $A \delta D$, we obtain a contradiction.

Remark 1.22. To show the proposed embedding of PSPROX into PSN, we use an additional property of $\left(\mathcal{B}^{X}, N_{\delta}\right)$.

Definition 1.23. We say that a pseudonear structure $\left(\mathcal{B}^{X}, M\right)$ and the space $\left(X, \mathcal{B}^{X}, M\right)$ are proxiform if they satisfy the following condition:

(px) If $\mathcal{S} \subset \underline{P} X, B \in \mathcal{B}^{X} \backslash\{\emptyset\}$ and for every $\mathcal{E} \subset\{B\} \cup\left(\mathcal{S} \cap \mathcal{B}^{X}\right)$ finite there exists $A, D \in \mathcal{B}^{X}$ with $\{A, D\} \in M(A) \cap M(D)$ such that $\left\{c l_{M}(E): E \in\right.$ $\mathcal{E}\} \ll\{A, D\}$, then $\mathcal{S} \in M(B)$.

By PX-PSN we denote the full subcategory of PSN, whose objects are proxiform pseudonear spaces.

Proposition 1.24. For a pseudoproximity space $\left(X, \mathcal{B}^{X}, \delta\right)$ the pseudonear space $\left(X, \mathcal{B}^{X}, N_{\delta}\right)$ is proxiform.

Proof. Without restriction, let for $B \in \mathcal{B} \backslash\{\emptyset\}, \mathcal{S} \notin N_{\delta}(B)$. Then, we can find $\mathcal{E} \subset\{B\} \cup\left(\mathcal{S} \cap \mathcal{B}^{X}\right)$ finite such that $\left\{c l_{\delta}(E): E \in \mathcal{E}\right\}<\nless\{A, D\}$ for every $A, D \in \mathcal{B}^{X}$ with $A \delta D$. Choose $E \in \mathcal{E}$ with $E \in \mathcal{S} \cap \mathcal{B}^{X}$ such that $c l_{\delta}(E) \nsupseteq A, D$ with $A \delta D$.

If $\mathcal{E} \in N_{\delta}(B)$, then $\{E\} \subset\{B\} \cup\left(\mathcal{E} \cap \mathcal{B}^{X}\right)$ finite implies $c l_{\delta}(E) \supset A$ or $c l_{\delta}(E) \supset$ $D$ for $A, D \in \mathcal{B}^{X}$ with $A \delta D$. But by the hypothesis, we obtain a contradiction.

Lemma 1.25. For a proxiform pseudonear space $\left(X, \mathcal{B}^{X}, M\right)$ the equation $N_{\gamma_{M}}=M$ holds.

Proof. To $\leq$ : For $B \in \mathcal{B}^{X} \backslash\{\emptyset\}$, let $\mathcal{S} \in N_{\gamma_{M}}(B)$ and $\mathcal{E} \subset\{B\} \cup\left(\mathcal{S} \cap \mathcal{B}^{X}\right)$ be finite, then $\left\{c l_{\gamma_{M}}(E): E \in \mathcal{E}\right\} \ll\{A, D\}$ with $A \gamma_{M} D$, hence $\{A, D\} \in M(A) \cap$ $M(D)$ follows. But $c l_{M}(E) \supset c l_{\gamma_{M}}(E)$ since $x \in c l_{\gamma_{M}}(E)$ implies $\{x\} \gamma_{M} E$, and 
$\{\{x\}, E\} \in M(\{x\}) \cap M(E)$ results. Consequently, $\{E\} \in M(\{x\})$ is valid, which implies $x \in c l_{M}(E)$. Since $\left(\mathcal{B}^{X}, M\right)$ is proxiform, $\mathcal{S} \in M(B)$ results.

To $\geq$ : Conversely, let $\mathcal{S} \in M(B)$. If $\mathcal{S} \notin N_{\gamma_{M}}(B)$, we can find $\mathcal{E} \subset\{B\} \cup\left(\mathcal{S} \cap \mathcal{B}^{X}\right)$ finite such that $\left\{c l_{\gamma_{M}}(E): E \in \mathcal{E}\right\}<\nless\{A, D\}$ for each $A, D \in \mathcal{B}^{X}$ with $A \gamma_{M} D$. Choose $E \in \mathcal{E}$ with $c l_{\gamma_{M}}(E) \nsupseteq B$. Hence, $E \in \mathcal{S} \cap \mathcal{B}^{X}$ follows (note that $E \subset$ $c l_{\gamma_{M}}(E)$ holds). $\{E\} \subset \mathcal{S}$ implies $\{E\} \in M(B)$ and since $\left(\mathcal{B}^{X}, M\right)$ is symmetric, $\{B, E\} \in M(B) \cap M(E)$ results, showing that $B \gamma_{M} E$ is valid. But by the supposition, $c l_{\gamma_{M}}(E) \nsupseteq E$, which leads to a contradiction.

Proposition 1.26. For a proxiform pseudonear space $\left(X, \mathcal{B}^{X}, M\right)$, the space $\left(X, \mathcal{B}^{X}, \gamma_{M}\right)$ forms a pseudoproximity space.

Proof. The axioms $\left(\mathrm{psp}_{1}\right)$ to $\left(\mathrm{psp}_{6}\right)$ are easy to verify. Now, to $\left(\operatorname{psp}_{7}\right)$, let $D \gamma_{M} c l_{\gamma_{M}}(B), B \in \mathcal{B}^{X}$ be given. By the definition, we get $\left\{D, c l_{\gamma_{M}}(B)\right\} \in$ $M(D) \cap M\left(c l_{\gamma_{M}}(B)\right)$. But $\left\{c l_{M}(D), c l_{M}(B)\right\} \ll\left\{D, c l_{\gamma_{M}}(B)\right\}$ implies

$$
\left\{c l_{M}(D), c l_{M}(B)\right\} \in M(D) \cap M\left(c_{\gamma_{M}}(B)\right) .
$$

Since $M(D) \cap M\left(c_{\gamma_{M}}(B)\right) \subset M(D) \cap M\left(c l_{M}(B)\right)=M(D) \cap M(B)$, note that $\left(\mathcal{B}^{X}, M\right)$ is symmetric, we get $\{D, B\} \in M(D) \cap M(B)$ because of density, and $D \gamma_{M} B$ results, which has to be shown.

Proposition 1.27. For pseudoproximity spaces $\left(X, \mathcal{B}^{X}, \delta^{X}\right),\left(Y, \mathcal{B}^{Y}, \delta^{Y}\right)$, let $f: X \longrightarrow Y$ be a function. Then, the following statements are equivalent:

(i) $f:\left(X, \mathcal{B}^{X}, \delta^{X}\right) \longrightarrow\left(Y, \mathcal{B}^{Y}, \delta^{Y}\right)$ is a bip-map;

(ii) $f:\left(X, \mathcal{B}^{X}, N_{\delta^{X}}\right) \longrightarrow\left(Y, \mathcal{B}^{Y}, N_{\delta^{Y}}\right)$ is a bin-map.

Proof. To (ii) $\Longrightarrow(\mathrm{i})$ : For $B_{1} \delta^{X} B_{2}$, let $\mathcal{E} \subset\left\{B_{1}\right\} \cup\left\{B_{1}, B_{2}\right\}$ be finite. Hence, $\left\{\operatorname{cl}_{\delta^{x}}(E): E \in \mathcal{E}\right\} \ll\left\{B_{1}, B_{2}\right\}$ and consequently, $\left\{B_{1}, B_{2}\right\} \in N_{\delta^{x}}\left(B_{1}\right)$ follows. Analogously, we get $\left\{B_{1}, B_{2}\right\} \in N_{\delta^{x}}\left(B_{2}\right)$ and both statements imply $\left\{B_{1}, B_{2}\right\} \in N_{\delta^{X}}\left(B_{1}\right) \cap N_{\delta^{X}}\left(B_{2}\right)$. By the hypothesis, $\left\{f\left[B_{1}\right], f\left[B_{2}\right]\right\}=f\left\{B_{1}, B_{2}\right\} \in$ $N_{\delta^{Y}}\left(f\left[B_{1}\right]\right) \cap N_{\delta^{Y}}\left(f\left[B_{2}\right]\right)$ is valid implying $f\left[B_{1}\right] \gamma_{N_{\delta} Y} f\left[B_{2}\right]$, which shows

$$
f\left[B_{1}\right] \delta^{Y} f\left[B_{2}\right]
$$

by the compatibility.

To (i) $\Longrightarrow$ (ii): Let for $B \in \mathcal{B}^{X} \backslash\{\emptyset\} \mathcal{S} \in N_{\delta^{X}}(B)$ and $\mathcal{E} \subset\{f[B]\} \cup\left(f \mathcal{S} \cap \mathcal{B}^{Y}\right)$ be finite. Our goal is to verify $\left\{c l_{\delta^{Y}}(E): E \in \mathcal{E}\right\} \ll\{A, D\}$ for some $A \delta^{Y} D$.

If $\left\{c l_{\delta^{Y}}(E): E \in \mathcal{E}<\nless\{A, D\}\right.$ for each $A, D$ with $A \delta D$, then choose $E \in \mathcal{E}$ with $E \in f \mathcal{S} \cap \mathcal{B}^{Y}$. Hence, $E=f[F]$ with $E \in \mathcal{B}^{Y}$ and $F \in \mathcal{S}$ implies $f^{-1}[E] \supset F$ with $F \in \mathcal{B}^{X}$ since $f$ is rebounded. But $\{F\} \subset\{B\} \cup\left(\mathcal{S} \cap \mathcal{B}^{X}\right)$ finite implies $c l_{\delta^{X}}(F) \supset\{\bar{A}, \bar{D}\}$ with $\bar{A} \delta^{X} \bar{D}$. If $c l_{\delta^{X}}(F) \supset \bar{A}$, then $c l_{\delta^{X}}(F) \delta^{X} \bar{D}$, which implies $F \delta^{X} \bar{D}$ and by the hypothesis, $f[F] \delta^{Y} f[\bar{D}]$ follows, and thus $E \delta^{Y} f[\bar{D}]$. But by the supposition, $c l_{\delta^{Y}}(E) \nsupseteq E$, which contradicts. The other case can then be handled analogously.

Theorem 1.28. The categories PSPROX and PX-PSN are isomorphic.

Proof. By using Proposition 1.21, Definition 1.23, Proposition 1.24, Lemma 1.25, 1.26 and Proposition 1.27, respectively. 
Another interesting property comes into play by considering finite set collections. This feature is also of importance for the examination of completions as we will see later.

Definition 1.29. A pseudonearness $\left(\mathcal{B}^{X}, N\right)$ and the triple $\left(X, \mathcal{B}^{X}, N\right)$ are called contiguous, provided $\left(\mathcal{B}^{X}, N\right)$ satisfies the following condition:

(ctg) $B \in \mathcal{B}^{X} \backslash\{\emptyset\}$ and $\mathcal{S} \notin N(B)$ implying the existence of $\mathcal{E} \subset\{B\} \cup \mathcal{S}$ finite with $\mathcal{E} \notin N(B)$.

Lemma 1.30. Every proxiform pseudonear space is contiguous.

Proof. Let a proxiform pseudonear space $\left(X, \mathcal{B}^{X}, M\right)$ be given such that for $B \in \mathcal{B}^{X} \backslash\{\emptyset\}, \mathcal{S} \notin M(B)$. Hence, by the hypothesis, we can find $\mathcal{E} \subset\{B\} \cup\left(\mathcal{S} \cap \mathcal{B}^{X}\right)$ finite such that $\left\{c l_{M}(E): E \in \mathcal{E}\right\}<\nless\{A, D\}, \forall A, D \in \mathcal{B}^{X}$ with $\{A, D\} \in M(A) \cap$ $M(D)$. Choose $E \in \mathcal{E}$ such that $c_{M}(E) \nsupseteq A, D$ with $\{A, D\} \in M(A) \cap M(D)$, $\forall A, D \in \mathcal{B}^{X}$. Hence, $\{E\} \subset\{B\} \cup\left(\mathcal{E} \cap \mathcal{B}^{X}\right)$ is finite, and for $A, D \in \mathcal{B}^{X}$ with $\{A, D\} \in M(A) \cap M(D)$, we have $c_{M}(E) \nsupseteq A, D$, which shows $\mathcal{E} \notin M(B)$.

Remark 1.31. On the other hand, if a saturated pseudonear space $\left(X, \mathcal{B}^{X}, M\right)$ is contiguous, then $\left(X, \eta_{M}\right)$ is a contigual nearness space, see [2] in connection with Lemma 1.10. In fact, let $\mathcal{S} \notin \eta_{M}$, then we can find $F \in \mathcal{S}$ with $\mathcal{S} \notin M(F)$. But $\left(\mathcal{B}^{X}, M\right)$ is contiguous, and thus, there exists $\mathcal{E} \subset\{F\} \cup \mathcal{S}$ finite with $\mathcal{E} \notin M(F)$. Hence, $\{F\} \cup \mathcal{E} \notin M(F)$ with $F \in\{F\} \cup \mathcal{E}$ and consequently, $\{F\} \cup \mathcal{E} \notin \eta_{M}$ follows with $\{F\} \cup \mathcal{E} \subset \mathcal{S}$, which shows $\eta_{M}$ is contigual.

Conversely, let $(X, \xi)$ be contigual. We have to show that $\left(\underline{P} X, N_{\xi}\right)$ is contiguous. For $B \in \underline{P} X \backslash\{\emptyset\}$, let $\mathcal{S} \notin N_{\xi}(B)$, then $\{B\} \cup \mathcal{S} \notin \xi$ implies the existence of $\mathcal{E} \subset\{B\} \cup \mathcal{S}$ finite with $\mathcal{E} \notin \xi$. Consequently, $\{B\} \cup \mathcal{E} \notin N_{\xi}(B)$ follows with $\{B\} \cup \mathcal{E} \subset\{B\} \cup \mathcal{S}$ finite and the claim results.

Definition 1.32. By C-PSN we denote the full subcategory of PSN, whose objects are contiguous pseudonear spaces.

Theorem 1.33. The category PX-PSN can be fully embedded into C-PSN (up to isomorphism) and, in the saturated case, the categories

\section{C-PSN and CONT,}

the category of contiguity spaces and contigual maps are isomorphic.

Proof. According to Theorem 1.28 and Lemma 1.30, respectively. Additionally, by using Remark 1.31 and Theorem 1.33 in connection with Theorem 1.13 and with respect to [2] and [1].

\section{THE B-COMPLETION}

An important property in the theory of uniform spaces is that of being complete. However, if a separated uniform space does not have this property, it can be densely embedded into a complete separated uniform space by a completion process such that the corresponding construction is universal. This is known as the Hausdorff completion of a separated uniform space. Herrlich [2] has extended this construction to a nearness space and obtained in the uniform case an equivalent result. At 


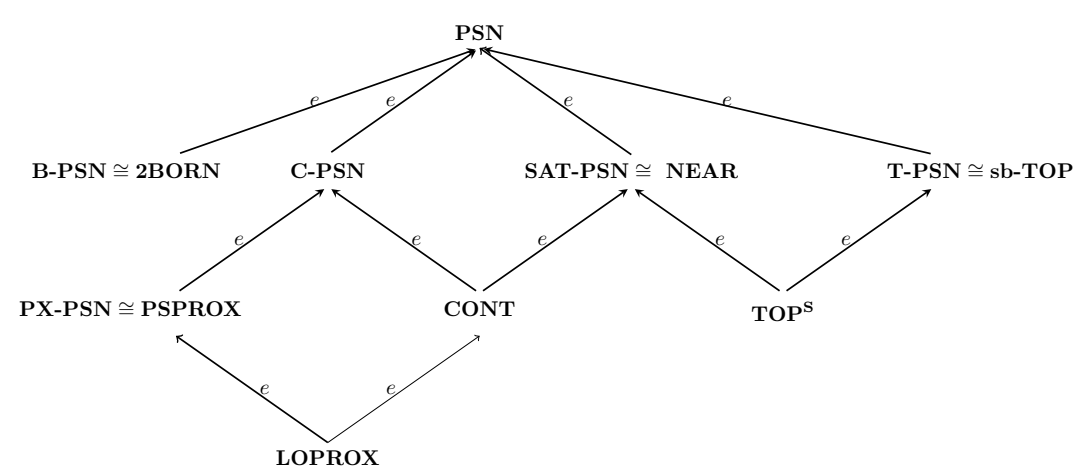

Figure 1. This diagram gives an overview on the connections between the existing categories, where $e$ denotes the corresponding embedding and $\cong$ isomorphisms.

this point, we find out that the definition of the completeness of a nearness space is closely related to the power set of its carrier set $X$ and thus more restricted than the one that will now be considered in the theory of pseudonearness. First, we will give a definition of an $N$-tape in a pseudonear space $\left(X, \mathcal{B}^{X}, N\right)$.

Definition 2.1. For a pseudonearness space $\left(X, \mathcal{B}^{X}, N\right), \mathcal{T} \subset \underline{P} X$ is called an $N$-tape in $\mathcal{B}^{X}$, provided it satisfies the following condition:

$\left(\operatorname{tp}_{1}\right) \mathcal{T} \in \underline{P} \mathcal{B}^{X} \cap N(B) \backslash\{\emptyset\}$ for some $B \in \mathcal{B} \backslash\{\emptyset\}$;

$\left(\operatorname{tp}_{2}\right) \quad A \in \mathcal{B}^{X}, D \in \mathcal{B}^{X} \backslash\{\emptyset\}$ and $\{A\} \cup \mathcal{T} \in N(D)$ implying $A \in \mathcal{T}$.

Lemma 2.2. For a nearness space $(X, \xi)$ and for $\mathcal{C} \subset \underline{P} X$ the following statements are equivalent:

(i) $\mathcal{C}$ is a $\xi$-cluster;

(ii) $\mathcal{C}$ is an $N_{\xi}$-tape in $\underline{P} X$.

Proof. To (i) $\Longrightarrow$ (ii): $\mathcal{C} \in \xi \backslash\{\emptyset\}$ implies the existence of $\emptyset \neq C \in \mathcal{C}$ and thus $\{C\} \cup \mathcal{C} \in \xi$, which implies $\mathcal{C} \in N_{\xi}(C) \backslash\{\emptyset\}$. Now, let $A \in \underline{P} X, D \in \underline{P} X \backslash\{\emptyset\}$ with $\{A\} \cup \mathcal{C} \in N_{\xi}(D)$. Consequently, $\{D\} \cup(\{A\} \cup \mathcal{C}) \in \xi$ implies $\{A\} \cup \mathcal{C} \in \xi$, and thus $A \in \mathcal{C}$ by the maximality of $\mathcal{C}$.

To (ii) $\Longrightarrow$ (i): Conversely, let $\mathcal{C} \in \xi \backslash\{\emptyset\}$ with $\mathcal{C} \subset \mathcal{A} \in \xi$. Then, for $A \in \mathcal{A}$, we get $\{A\} \cup \mathcal{C} \in \xi$, which implies $\mathcal{C} \in N_{\xi}(A)$, and by the symmetry of $N_{\xi}$, we obtain $\{A\} \cup \mathcal{C} \in N_{\xi}(A)$. Hence, $A \in \mathcal{C}$ according to $\left(\operatorname{tp}_{2}\right)$, and the claim follows.

Example 2.3. For a pseudonear space $\left(X, \mathcal{B}^{X}, N\right)$ and for each $x \in X, \mathcal{T}_{x}^{N}:=$ $\left\{A \in \underline{P B^{X}}:\{A\} \in N(\{x\})\right\}$ is an N-tape in $\mathcal{B}^{X}$. In fact, let $A \in \mathcal{T}_{x}^{N}$. Hence, $\{A\} \in N(\{x\})$, which is equivalent to $x \in c l_{N}(A)$. But then, $\left\{c l_{N}(A): A \in\right.$ $\left.\mathcal{T}_{x}^{N}\right\} \ll\{\{x\}\} \in N(\{x\})$ and consequently, $\left\{c l_{N}(A): A \in \mathcal{T}_{x}^{N}\right\} \in N(\{x\})$ implies $\mathcal{T}_{x}^{N} \in N(\{x\})$, thus (tp $\left.{ }_{1}\right)$ is fulfilled. Finally, $B \in \mathcal{B}^{X}$ and $D \in \mathcal{B}^{X} \backslash\{\emptyset\},\{B\} \cup$ $\mathcal{T}_{x}^{N} \in N(D)$. Hence, by the symmetry, we get $\{D\} \cup\left(\{B\} \cup \mathcal{T}_{x}^{N}\right) \in N(B)$, which implies $\mathcal{T}_{x}^{N} \in N(B)$. By applying the symmetry again, we obtain $\{B\} \cup \mathcal{T}_{x}^{N} \in$ $N(\{x\})$. Note that $\{x\} \in \mathcal{T}_{x}^{N}$ is valid. Consequently, $\{B\} \in N(\{x\})$ follows, which shows $B \in \mathcal{T}_{x}^{N}$.

Definition 2.4. A pseudonear space $\left(X, \mathcal{B}^{X}, N\right)$ is called $b$-complete, provided $\left(\mathcal{B}^{X}, N\right)$ satisfies the following condition: 
(b-cpl) $\forall \mathcal{T} \subset \underline{P} X$ N-tape in $\mathcal{B}^{X} \exists x \in X$ such that $\{x\} \in \mathcal{T}$.

Remark 2.5. According to the definition of completeness in a nearness space [2] we point out that, in the saturated case, the terms b-complete and complete coincide. Further, we note that every non-empty finite pseudonear space is already b-complete. Moreover, we infer that a uniform space is complete as a uniform space iff its associated saturated pseudonear space is b-complete [2]. Here, for a uniform space $(X, \mathcal{U})$, where $\mathcal{U}$ is regarded as diagonal uniformity, the associated saturated pseudonearness $\left(\underline{P} X, N_{\mathcal{U}}\right)$ is defined by setting:

$$
N_{\mathcal{U}}(\emptyset):=\{\emptyset\}
$$

and

$$
N_{\mathcal{U}}(B):=\{\mathcal{S} \subset \underline{P} X: \forall R \in \mathcal{U} \cap\{R(F): F \in \mathcal{F} \in \mathcal{S} \cup\{B\}\} \neq \emptyset\}
$$

for each $B \in \underline{P} X \backslash\{\emptyset\}$. Furthermore, we note that $\left(\underline{P} X, N_{\mathcal{U}}\right)$ satisfies the condition for being a uniform pseudonearness $(\underline{P} X, M)$, i.e.

(U) $B \in \underline{P} X \backslash\{\emptyset\}$ and $\mathcal{S} \notin M(B)$ implying $\exists \mathcal{A} \notin M(B) \forall x \in B \exists F \in \mathcal{S}$, $F \subset \cap\{A \in \mathcal{A}: x \notin A\}$.

As already mentioned, we know that every separated uniform space has a corresponding completion. In the following, we will see that the completion can also be obtained as a completion of its associated pseudonear space up to isomorphism.

Lemma 2.6. (i) Every bornoform pseudonear space is b-complete;

(ii) Every topoform pseudonear space is b-complete.

Proof. By straight-forward execution.

Now, according to [2], we will note some details for the construction of a bcompletion of a pseudonear space.

Theorem 2.7. Let $\left(X, \mathcal{B}^{X}, N\right)$ be a pseudonear space. Then, we consider the triple $\left(X^{*}, \mathcal{B}^{X^{*}}, N^{*}\right)$, where $X^{*}:=\left\{\mathcal{T} \subset \underline{P} X: \mathcal{T}\right.$ is an $N$-tape in $\left.\mathcal{B}^{X}\right\} ; \mathcal{B}^{X^{*}}:=$ $\left\{B^{*} \subset X^{*}: \exists D \in \mathcal{B}^{X} \forall \mathcal{T} \in B^{*}, \mathcal{T} \in N(D)\right\}$ and $N^{*}: \mathcal{B}^{X^{*}} \longrightarrow \underline{P}\left(\underline{P}\left(\underline{P} X^{*}\right)\right)$ is defined by setting:

$$
N^{*}(\emptyset):=\{\emptyset\}
$$

and

$$
\begin{aligned}
N^{*}\left(B^{*}\right):= & \left\{\mathcal{A}^{*} \subset \underline{P} X^{*}: \exists B \in \mathcal{B}^{X} \backslash\{\emptyset\}\left\{F \in \mathcal{B}^{X}: \exists A^{*} \in\left(\mathcal{A}^{*} \cap \mathcal{B}^{X^{*}}\right)\right.\right. \\
& \left.\left.\cup\left\{B^{*}\right\}, F \in \Delta A^{*}\right\} \in N(B)\right\} \text { for each } B^{*} \in \mathcal{B}^{X^{*} \backslash\{\emptyset\},} \\
& \text { where for } A^{*} \subset X^{*}, \Delta A^{*}:=\left\{A \in \mathcal{B}^{X}: \forall \mathcal{T} \in A^{*}, A \in \mathcal{T}\right\} .
\end{aligned}
$$

Then, $\left(X^{*}, \mathcal{B}^{X^{*}}, N^{*}\right)$ is a separated b-complete pseudonear space such that

$$
c l_{N^{*}}(j[X])=X^{*},
$$

where $j: X \longrightarrow X^{*}$ denotes the function assigning the $N$-tape $\mathcal{T}_{x}^{N}$ to each $x \in X$.

Remark 2.8. In this context, we note that a pseudonear space $\left(X, \mathcal{B}^{X}, N\right)$ is called separated, provided $\left(\mathcal{B}^{X}, N\right)$ satisfies

(sep) $x, z \in X$ and $\{\{x\}\} \in N(\{z\})$ implying $x=z$. 
Note that $\left(\mathcal{B}^{X}, N^{b^{X}}\right)$ in 1.4 is especially separated.

Proof. First, we take into account that $\left(\mathcal{B}^{X^{*}}, N^{*}\right)$ defines a pseudonearness. $\left(X^{*}, \mathcal{B}^{X^{*}}, N^{*}\right)$ is b-complete. To this end, let $\mathcal{T}^{*}$ be an $N^{*}$-tape in $\mathcal{B}^{X^{*}}$. Then, $\mathcal{T}^{*} \in \underline{P} \mathcal{B}^{X^{*}} \cap N^{*}\left(B^{*}\right) \backslash\{\emptyset\}$ for some $B^{*} \in \mathcal{B}^{X^{*}} \backslash\{\emptyset\}$. By the definition, we can find $B \in \mathcal{B}^{X} \backslash\{\emptyset\}$ such that $\mathcal{D}:=\left\{F \in \mathcal{B}^{X}: \exists A^{*} \in\left(\mathcal{T}^{*} \cap \mathcal{B}^{X^{*}}\right) \cup\left\{B^{*}\right\}, F \in \Delta A^{*}\right\} \in$ $N(B)$. By the symmetry, $\{B\} \cup \mathcal{D}=: \mathcal{T} \in \underline{P} \mathcal{B}^{X} \cap N(B) \backslash\{\emptyset\}$, so it remains to verify that $\mathcal{T}$ satisfies $\left(\mathrm{tp}_{2}\right)$. So, let $A \in \mathcal{B}^{X}, \bar{D} \in \mathcal{B}^{X} \backslash\{\emptyset\}$ with $\{A\} \cup \mathcal{T} \in N(D)$. The goal is $A \in \mathcal{T}$. We have $\Delta j[A] \cup \mathcal{T} \in N(D)$ since $\left\{c l_{N}(F): F \in \Delta j[A] \cup \mathcal{T}\right\} \ll$ $\{A\} \cup \mathcal{T}$. Note that $A \subset c_{N}(F)$ is valid. Because of $j[A] \in \mathcal{B}^{X^{*}},\{j[A]\} \cup \mathcal{T}^{*} \in$ $N^{*}\left(B^{*}\right)$ follows since $\mathcal{V}:=\left\{F \in \mathcal{B}^{X}: \exists D^{*} \in\left(\left(\{j[A]\} \cup \mathcal{T}^{*}\right) \cap \mathcal{B}^{X^{*}}\right) \cup\left\{B^{*}\right\}, F \in\right.$ $\left.\Delta D^{*}\right\} \subset \Delta j[A] \cup \mathcal{T}$. But by the hypothesis, $\mathcal{T}^{*}$ is an $N^{*}$-tape in $\mathcal{B}^{X^{*}}$. Thus, $j[A] \in \mathcal{T}^{*}$. Furthermore, $j[A] \in\left(\mathcal{T}^{*} \cap \mathcal{B}^{X^{*}}\right) \cup\left\{B^{*}\right\}$ implies $\Delta j[A] \subset \mathcal{T}$ and since $A \in \Delta j[A]$ holds, $A \in \mathcal{T}$ results. Now, $\Delta\{\mathcal{T}\}=\mathcal{T}$ implies $\mathcal{T} \cup \Delta\{\mathcal{T}\} \in N(B)$. Thus, $\{\{\mathcal{T}\}\} \cup \mathcal{T}^{*} \in N^{*}\left(B^{*}\right)$ can be deduced, which implies $\{\mathcal{T}\} \in \mathcal{T}^{*}$ since, by the hypothesis, $\mathcal{T}^{*}$ is an $N^{*}$-tape in $\mathcal{B}^{X^{*}}$. Consequently, $\left(X^{*}, \mathcal{B}^{X^{*}}, N^{*}\right)$ is b-complete. Next, we are showing $\left(X^{*}, \mathcal{B}^{X^{*}}, N^{*}\right)$ is separated. To this end, let $\mathcal{C}, \mathcal{D} \in X^{*}$ with $\{\{\mathcal{C}\}\} \in N^{*}(\{\mathcal{D}\})$, then we can find $B \in \mathcal{B}^{X} \backslash\{\emptyset\}$ such that $\mathcal{V}:=\left\{F \in \mathcal{B}^{X}: \exists A^{*} \in\left(\{\{\mathcal{C}\}\} \cap \mathcal{B}^{X^{*}}\right) \cup\left\{B^{*}\right\}, F \in \Delta A^{*}\right\} \in N(B)$. Consequently, $\mathcal{C} \cup \mathcal{D} \in N(B)$ results. It remains to show that the inclusion $\mathcal{D} \subset \mathcal{C}$ holds. $F \in \mathcal{D}$ implies $\{F\} \cup \mathcal{C} \in N(B)$ and by applying $\left(\operatorname{tp}_{2}\right), F \in \mathcal{C}$ is valid.

Now, we will show that $c l_{X^{*}}(j[X])=X^{*}$ can be deduced. So, let $\mathcal{T} \in X^{*}$. Hence, $\mathcal{T} \in \underline{P \mathcal{B}^{X}} \cap N(B) \backslash\{\emptyset\}$ for some $B \in \mathcal{B}^{X} \backslash\{\emptyset\}$. It remains to prove $\mathcal{T} \in$ $c l_{X^{*}}(j[B])$, which means $\{j[B]\} \in N^{*}(\{\mathcal{T}\})$. To this end, it suffices to verify that $\mathcal{V}:=\left\{F \in \mathcal{B}^{X}: \exists A^{*} \in\left(\{j[B]\} \cap \mathcal{B}^{X^{*}}\right) \cup\{\{\mathcal{T}\}\}, F \in \Delta A^{*}\right\} \in N(B)$ holds. To show that $\mathcal{V} \subset \mathcal{T}$ is valid, let $F \in \mathcal{V}$, then $F \in \Delta j[B] \cup \mathcal{T}$ follows, which implies $B \subset c l_{N}(F)$.

By the symmetry of $\left(\mathcal{B}^{X}, N\right)$, we have $\{B\} \cup \mathcal{T} \in N(B)$ and consequently, $\left\{c l_{N}(F)\right\} \cup\left\{c l_{N}(A): A \in \mathcal{T}\right\} \in N(B)$ results. Thus, $\{F\} \cup \mathcal{T} \in N(B)$, since $\left(\mathcal{B}^{X}, N\right)$ is dense and $F \in \mathcal{T}$ concludes the proof by applying $\left(\operatorname{tp}_{2}\right)$.

Proposition 2.9. For a pseudonear space $\left(X, \mathcal{B}^{X}, N\right), j:\left(X, \mathcal{B}^{X}, N\right) \longrightarrow$ $\left(X^{*}, \mathcal{B}^{X^{*}}, N^{*}\right)$ is a bin-map and for each $B \in \mathcal{B}^{X} \backslash\{\emptyset\}$, the following statements are equivalent:

(i) $\mathcal{A} \in N(B)$;

(ii) $j \mathcal{A} \in N^{*}(j[B])$.

Proof. Evidently, for each $B \in \mathcal{B}^{X}, j[B] \in \mathcal{B}^{X^{*}}$ is valid. On the other hand, $j$ is rebounded. Let $B^{*} \in \mathcal{B}^{X^{*}}$. Hence, we can find $D \in \mathcal{B}^{X}$ such that $\forall \mathcal{T} \in$ $B^{*} \mathcal{T} \in N(D)$ is valid. We will show that $j^{-1}\left[B^{*}\right] \subset c l_{N}(D)$ can be deduced. So, let $x \in j^{-1}\left[B^{*}\right]$. Hence, $j(x) \in B^{*}$, which implies $j(x) \in N(D)$ by applying the hypothesis. Thus, $D \neq \emptyset$. By the symmetry, we get $\{D\} \cup j(x) \in N(D)$. But $j(x)$ is an $\mathrm{N}$-tape in $\mathcal{B}^{X}$. Hence, $D \in j(x)$ follows, which shows $x \in c l_{N}(D)$.

Now, let $\mathcal{A}$ be an element of $N(B)$ for some $B \in \mathcal{B}^{X} \backslash\{\emptyset\}$. We will show that $\mathcal{V}:=\left\{F \in \mathcal{B}^{X}: \exists A^{*} \in\left(j \mathcal{A} \cap \mathcal{B}^{X^{*}}\right) \cup\{j[B]\}, F \in \Delta A^{*}\right\} \in N(B)$ is true. By the symmetry, we get $\{B\} \cup \mathcal{A} \in N(B)$. It remains to verify that 
$m:=\left\{c l_{N}(F): F \in \mathcal{V}\right\} \ll\{B\} \cup \mathcal{A} . D \in m$ implies $D=c_{N}(F)$ for some $F \in \mathcal{V}$. If $F \in \Delta j[B]$, then $B \subset c l_{N}(F)=D$ follows and the claim results.

Now, if $F \in \Delta A^{*}$ for some $A^{*} \in j \mathcal{A} \cap \mathcal{B}^{X^{*}}$, then $A^{*}=j[A]$ for some $A \in$ $\mathcal{A} \cap \mathcal{B}^{X}$. Note that $j^{-1}[j[A]] \in \mathcal{B}^{X}$ is valid with $j^{-1}[j[A]] \supset A$. Consequently, $A \subset c l_{N}(F)=D$ results, which shows the claim. Conversely, let $B \in \mathcal{B}^{X} \backslash\{\emptyset\}$ and $\mathcal{A} \subset \underline{P} X$ with $j \mathcal{A} \in N^{*}(j[B])$. Then, we can find $D \in \mathcal{B}^{X} \backslash\{\emptyset\}$ such that $\mathcal{V}:=\left\{F \in \mathcal{B}^{X}: \exists A^{*} \in\left(j \mathcal{A} \cap \mathcal{B}^{X^{*}}\right) \cup\{j[B]\}, F \in \Delta A^{*}\right\} \in N(D)$. Our goal is to show $\mathcal{A} \in N(B)$. Therefore, it suffices to verify $\mathcal{A} \cap \mathcal{B}^{X} \in N(B)$. Since $\{B\} \in \mathcal{V}$ and $\left(\mathcal{B}^{X}, N\right)$ is symmetric, $\{D\} \cup \mathcal{V} \in N(B)$ follows. We are showing $\mathcal{A} \cap \mathcal{B}^{X} \subset \mathcal{V}$. $A \in \mathcal{A} \cap \mathcal{B}^{X}$ implies $j[A] \in\left(j \mathcal{A} \cap \mathcal{B}^{X^{*}}\right) \cup\{j[B]\}$ with $A \in \Delta j[A]$. Hence, $A \in \mathcal{V}$ follows. Thus, $\mathcal{A} \in N(B)$ since $\left(\mathcal{B}^{X}, N\right)$ is b-absorbed.

Lemma 2.10. For a pseudonear space $\left(X, \mathcal{B}^{X}, N\right)$ each successive pair of conditions are equivalent:

(i) $j$ is injective;

(ii) $\left(X, \mathcal{B}^{X}, N\right)$ is separated;

(iii) $j$ is surjective;

(iv) $\left(X, \mathcal{B}^{X}, N\right)$ is b-complete.

Proof. To (i) $\Longrightarrow$ (ii): Let $x, z \in X$ with $\{\{x\}\} \in N(\{z\})$ and suppose $x \neq z$. Then, $j(x) \neq j(z)$ by applying the hypothesis. Without restriction, choose $F \in$ $j(x)$ with $F \neq j(z)$. Thus, $x \in c l_{N}(F)$ implies $\left\{c l_{N}(F)\right\} \in N(\{z\})$. Consequently, $\{F\} \in N(\{z\})$ follows, showing that $F \in j(z)$ is valid, which contradicts.

To (ii) $\Longrightarrow$ (i): Now, let $x, z \in X$ with $j(x)=j(z)$. Hence, $\{x\} \in j(z)$ follows, which implies $\{\{x\}\} \in N(\{z\})$. But by the hypothesis, $x=z$ is valid, which shows the claim.

To (iii) $\Longrightarrow$ (iv): Let $j$ be surjective and $\mathcal{T} \subset \underline{P} X$ an $\mathrm{N}$-tape in $\mathcal{B}^{X}$. Since $\mathcal{T} \in X^{*}$, we can find $x \in X$ with $j(x)=\mathcal{T}$. But $\{x\} \in j(x)$ implies the claim.

To (iv) $\Longrightarrow$ (iii): Let $\mathcal{T} \in X^{*}$, then $\mathcal{T}$ is an N-tape in $\mathcal{B}^{X}$ and by the hypothesis, we can find $x \in X$ such that $\{x\} \in \mathcal{T}$. Since $\mathcal{T} \in \underline{P \mathcal{B}^{X}} \cap N(B) \backslash\{\emptyset\}$ for some $B \in \mathcal{B}^{X} \backslash\{\emptyset\}$ and $\left(\mathcal{B}^{X}, N\right)$ is symmetric, $\{B\} \cup \mathcal{T} \in N(\{x\})$ is valid. Now, for $F \in \mathcal{T}$, we have $\{F\} \in N(\{x\})$ and $F \in j(x)$ follows, showing that $\mathcal{T} \subset j(x)$. On the other hand, $F \in j(x)$ implies $x \in c l_{N}(F)$ with $c l_{N}(F) \in \mathcal{B}^{X}$. Hence, $\left\{c l_{N}(F)\right\} \cup\left\{c l_{N}(A): A \in \mathcal{T}\right\} \ll \mathcal{T} \in N(B)$. Consequently, $\{F\} \cup N(B)$ results, showing that $F \in \mathcal{T}$ is valid by applying $\left(\mathrm{tp}_{2}\right)$.

Remark 2.11. Now, it is interesting to note that if $\left(X, \mathcal{B}^{X}, N\right)$ is a saturated pseudonear space, in other words representing a nearness space, the space $\left(X^{*}, \mathcal{B}^{X^{*}}, N^{*}\right)$ is saturated, too. In fact, $X^{*} \in \mathcal{B}^{X^{*}}$ because $X \in \mathcal{B}^{X}$ is valid and $\left(\mathcal{B}^{X}, N\right)$ is especially isotone, meaning that $\emptyset \neq B_{1} \subset B \in \mathcal{B}^{X}$ implies $N\left(B_{1}\right) \subset N(B)$. Furthermore, let $(X, \xi)$ be a nearness space, then we consider the pseudonear space $\left(X, \underline{P} X, N_{\xi}\right)$. If $f:(X, \xi) \longrightarrow\left(X^{*}, \xi^{*}\right)$ denotes the Herrlichcompletion of $(X, \xi),[2]$, then $N_{\xi}^{*}=N_{\xi^{*}}$. In this context, we refer to Remark 1.7, Lemma 2.2, and Remark 2.5, respectively. Thus, the above mentioned equation delivers the expected completion and represents the Herrlich-completion up to isomorphism. In fact, let $B^{*} \in \underline{P} X^{*} \backslash\{\emptyset\}$ and $\mathcal{A}^{*} \in N_{\xi^{*}}\left(B^{*}\right)$. Our goal is to verify $\left\{B^{*}\right\} \cup \mathcal{A}^{*} \in \xi^{*}$, which means $\xi\left(\cup\left\{\cap W: W \in\left\{B^{*}\right\} \cup \mathcal{A}^{*}\right\}\right)$. By the hypothesis, we can find $B \in \underline{P} X \backslash\{\emptyset\}$ with $\mathcal{V}:=\left\{F \in \underline{P} X: \exists A^{*} \in \mathcal{A}^{*} \cup\left\{B^{*}\right\}\right.$, 
$\left.F \in \Delta A^{*}\right\} \in N_{\xi}(B)$. Hence, $\{B\} \cup \mathcal{V} \in \xi$ follows. It remains to prove $\cup\{\cap W$ : $\left.W \in\left\{B^{*}\right\} \cup \mathcal{A}^{*}\right\} \subset\{B\} \cup \mathcal{V}$. Now, $F \in \cup\left\{\cap W: W \in\left\{B^{*}\right\} \cup \mathcal{A}^{*}\right\}$ implies the existence of $W \in\left\{B^{*}\right\} \cup \mathcal{A}^{*}$ with $F \in \cap W$. If $W=B^{*}$, then $F \in \Delta B^{*}$ follows, implying $F \in \mathcal{V} \cup\{B\}$. If $W \in A^{*}$, we have $F \in \Delta W$, and thus $F \in \mathcal{V} \cup\{B\}$. Conversely, let $A^{*} \in N_{\xi^{*}}\left(B^{*}\right)$. Hence, $\left\{B^{*}\right\} \cup \mathcal{A}^{*} \in \xi^{*}$ follows, implying $\xi\left(m:=\cup\left\{\cap W: W \in\left\{B^{*}\right\} \cup \mathcal{A}^{*}\right\}\right)$. We can choose $B \in \underline{P} X \forall \mathcal{T} \in B^{*}, B \in \mathcal{T}$. Our goal is $\{B\} \cup \mathcal{V}:=\left\{F \in \underline{P} X: \exists A^{*} \in \mathcal{A}^{*} \cup\left\{B^{*}\right\}, F \in \Delta A^{*}\right\} \subset m$. So, let $A \in\{B\} \cup \mathcal{V}$ and verify $A=B$. We put $W=B^{*}$. Hence, $A \in \cap W$ follows. Secondly, let $A \in \mathcal{V}$, then there exists $A^{*} \in \mathcal{A}^{*} \cup\left\{B^{*}\right\}$ with $F \in \Delta A^{*}$. If $A^{*}=B^{*}$, then we also put $W:=B^{*}$ and the claim follows. Finally, let $A^{*} \in \mathcal{A}^{*}$, then by setting $W:=\mathcal{A}^{*}, F \in \cap W$ results.

As an important résumé, we can now infer that a saturated pseudonear space is uniform iff its b-completion is uniform (compare also with Remark 2.5). Thus, the associated uniform space is complete as a uniform space. Further, we are looking in more detail at those properties of pseudonear spaces which were carried over by the previously defined completion process. First, we note in connection with Definition 1.29 that every non-empty finite pseudonear space is contiguous.

Proposition 2.12. For a separated pseudonear space $\left(X, \mathcal{B}^{X}, N\right)$ the following statements are equivalent;

(i) $\left(X, \mathcal{B}^{X}, N\right)$ is contiguous;

(ii) $\left(X^{*}, \mathcal{B}^{X^{*}}, N^{*}\right)$ is contiguous.

Proof. To (i) $\Longrightarrow$ (ii): Let $B^{*} \in \mathcal{B}^{X^{*}} \backslash\{\emptyset\}$ and $\mathcal{A} \notin N^{*}\left(B^{*}\right)$. Hence, by the definition, we can find $D \in \mathcal{B}^{X}$ such that $\forall \mathcal{T} \in B^{*}, \mathcal{T} \in N(D)$. Since $D \neq$ $\emptyset$, we get $\mathcal{V}:=\left\{F \in \mathcal{B}^{X}: \exists A^{*} \in\left(\mathcal{A}^{*} \cap \mathcal{B}^{X^{*}}\right) \cup\left\{B^{*}\right\}, F \in \Delta A^{*}\right\} \notin N(D)$. Otherwise, if $D=\emptyset$, choose $\mathcal{T} \in B^{*}$ with $\mathcal{T} \in N(\emptyset)$. Hence, $\mathcal{T}=\emptyset$ follows. But $\mathcal{T} \in \underline{P B}^{X} \cap N(B) \backslash\{\emptyset\}$ for some $B \in \mathcal{B}^{X} \backslash\{\emptyset\}$ and since $\left(\mathcal{B}^{X}, N\right)$ is symmetric, $\{B\} \cup \mathcal{T} \in N(B)$ results. By applying $\left(\operatorname{tp}_{2}\right), B \in \mathcal{T}$ is valid, which contradicts. Since, by the hypothesis, $\left(\mathcal{B}^{X}, N\right)$ is contiguous, we can find $\mathcal{E} \subset\{D\} \cup \mathcal{V}$ finite with $\mathcal{E} \notin N(D)$. Now, we put $\mathcal{E}^{*}:=\left\{D^{*} \in \mathcal{A}^{*}: D^{*}=j[E]\right.$ for some $\left.E \in \mathcal{E}\right\}$. Thus, $\mathcal{E}^{*} \subset\left\{B^{*}\right\} \cup \mathcal{A}^{*}$ is finite. Our goal is to verify $\mathcal{E}^{*} \notin N^{*}\left(B^{*}\right)$. If $\mathcal{E}^{*} \in N^{*}\left(B^{*}\right)$, we can find $B \in \mathcal{B}^{X} \backslash\{\emptyset\}$ such that $m:=\left\{E \in \mathcal{B}^{X}: \exists D^{*} \in\left(\mathcal{E}^{*} \cap \mathcal{B}^{X^{*}}\right) \cup\left\{B^{*}\right\}, E \in\right.$ $\left.\Delta D^{*}\right\} \in N(B)$. But $D \in m$ is valid because, by choosing $B^{*} \in\left(\mathcal{E}^{*} \cap \mathcal{B}^{X^{*}}\right) \cup\left\{B^{*}\right\}$, we have $D \in \Delta B^{*}$. In fact, for each $\mathcal{T} \in B^{*}, \mathcal{T} \in N(D)$ implies $D \in \mathcal{T}$. This holds by the symmetry of $\left(\mathcal{B}^{X}, N\right)$ and by applying $\left(\operatorname{tp}_{2}\right)$. Consequently, $\{B\} \cup m \in N(D)$ follows and $m \in N(D)$ results. But $\mathcal{E} \subset m$ contradicts. In fact, let $E \in \mathcal{E}$. Hence, $D^{*}=j[E] \in\left(\mathcal{E}^{*} \cap \mathcal{B}^{X^{*}}\right) \cup\left\{B^{*}\right\}$. Note also that $E \in \mathcal{B}^{X}$ and $j$ is bounded. Now, let $\mathcal{D} \in D^{*}$. Hence, $\mathcal{D}=j(x)$ for some $x \in E$ and consequently, $\mathcal{D}=\mathcal{T}_{x}^{N}$. But $x \in \operatorname{cl}_{N}(E)$ implies $E \in \mathcal{D}$, which has to be shown.

To (ii) $\Longrightarrow$ (i): For $B \in \mathcal{B}^{X} \backslash\{\emptyset\}$, let $\mathcal{S} \notin N(B)$. Hence, $j \mathcal{S} \notin N^{*}(j[B])$ by applying Proposition 2.9. By the hypothesis, we can find $\mathcal{E}^{*} \subset\{j[B]\} \cup j \mathcal{S}$ finite with $\mathcal{E}^{*} \notin N^{*}(j[B])$. Consequently, $\mathcal{V}:=\left\{F \in \mathcal{B}^{X}: \exists D^{*} \in\left(\mathcal{E}^{*} \cap \mathcal{B}^{X^{*}}\right) \cup\left\{B^{*}\right\}\right.$, $\left.F \in \Delta D^{*}\right\} \notin N(B)$. We put $\mathcal{E}:=\left\{j^{-1}\left[D^{*}\right] D^{*} \in \mathcal{E}^{*}\right\}$. Hence, $\mathcal{E} \subset\{B\} \cup \mathcal{S}$ is finite because $A \in \mathcal{E}$ implies $A=j^{-1}\left[D^{*}\right]$ for some $D^{*} \in \mathcal{E}^{*}$. Thus, $D^{*}=j[D]$ for some $D \in\{B\} \cup \mathcal{S}$. Hence, $A=j^{-1}\left[D^{*}\right]=j^{-1}[j[D]]=D$ follows since $j$ is injective, see 2.10. If $\mathcal{E} \in N(B)$, then we will show that $\left\{c l_{N}(F): F \in \mathcal{V}\right\} \ll \mathcal{E}$, which 
leads to a contradiction. $D \in\left\{c l_{N}(F): F \in \mathcal{V}\right\}$ implies $D=c l_{N}(F)$ for some $F \in \mathcal{V}$. So we can find $D^{*} \in\left(\mathcal{E}^{*} \cap \mathcal{B}^{X^{*}}\right) \cup\{j[B]\}$ with $F \in \Delta D^{*}$ and consequently, $j^{-1}\left[D^{*}\right] \in \mathcal{E}$ follows. $D \supset j^{-1}\left[D^{*}\right]$ since $x \in j^{-1}\left[D^{*}\right]$ implies $j(x) \in D^{*}$. Hence, $F \in j(x)=\mathcal{T}_{x}^{N}$ and $x \in c l_{N}(F)=D$ results. Thus, $\left\{c l_{N}(F): F \in \mathcal{V}\right\} \in N(B)$ implies $\mathcal{V} \in N(B)$, which contradicts and the claim is true.

A slight modification to the term contiguous leads to the following definition.

Definition 2.13. A pseudonear space $\left(X, \mathcal{B}^{X}, N\right)$ is called full-bounded iff it satisfies the following equivalent conditions:

$\left(\mathrm{fbd}_{1}\right) \mathcal{F} \in F I L(X) \backslash\{\underline{P} X\}$ implies $\mathcal{F} \in \cap\left\{N(F): F \in \mathcal{F} \cap \mathcal{B}^{X}\right\}$;

$\left(\mathrm{fbd}_{2}\right) B \in \mathcal{B}^{X} \backslash\{\emptyset\}$ and $\mathcal{S} \notin N(B)$ implies the existence of $\mathcal{E} \subset\{B\} \cup \mathcal{S}$ finite with $B \cap(\cap \mathcal{E})=\emptyset$.

Remark 2.14. First, let us note that every contiguous pseudonear space is full-bounded. In fact, for $B \in \mathcal{B}^{X} \backslash\{\emptyset\}$, let $\mathcal{S} \notin N(B)$. Hence, by the hypothesis, we can find $\mathcal{E} \subset\{B\} \cup \mathcal{S}$ finite with $\mathcal{E} \notin N(B)$. If $B \cap(\cap \mathcal{E}) \neq \emptyset$, then there exists $x \in B$ with $x \in \cap \mathcal{E}$. But $\mathcal{E} \in N(\{x\}) \subset N(B)$ contradicts.

Now, let $\left(X, \mathcal{B}^{X}, N\right)$ satisfy $\left(\mathrm{fbd}_{2}\right)$ and, for $\mathcal{F} \in F I L(X) \backslash\{\underline{P} X\}$, suppose $\mathcal{F} \notin$ $\cap\left\{N(F): F \in \mathcal{F} \cap \mathcal{B}^{X}\right\}$. Hence, we can find $F \in \mathcal{F} \cap \mathcal{B}^{X}$ with $\mathcal{F} \notin N(F)$. By applying the hypothesis there is some $\mathcal{E} \subset\{F\} \cup \mathcal{F}=\mathcal{F}$ finite with $F \cap(\cap \mathcal{E})=\emptyset$, which contradicts. Note that $\mathcal{F}$ is a proper filter. Conversely, let $\left(X, \mathcal{B}^{X}, N\right)$ satisfy $\left(\mathrm{fbd}_{1}\right)$. Let $B \in \mathcal{B}^{X} \backslash\{\emptyset\}$ and $\mathcal{S} \notin N(B)$ and suppose $B \cap(\cap \mathcal{E}) \neq \emptyset$ for every $\mathcal{E} \subset\{B\} \cup \mathcal{S}$ finite. We put $\mathcal{F}:=\{F \subset X: \exists \mathcal{E} \subset\{B\} \cup \mathcal{S}$ finite $F \supset \cap \mathcal{E}\}$. Then, $\mathcal{F} \in F I L(X) \backslash\{\underline{P} X\}$. Evidently, $B \in \mathcal{F}$ and $\mathcal{F} \neq \underline{P} X$. Furthermore, $\mathcal{F}$ is closed up to supersets. Now, let $F_{1}, F_{2} \in \mathcal{F}$. Then, we can find $\mathcal{E}_{1} \supset\{B\} \cup \mathcal{S}$ finite and $\mathcal{E}_{2} \supset\{B\} \cup \mathcal{S}$ finite with $F_{1} \supset \cap \mathcal{E}_{1}$ and $F_{2} \supset \cap \mathcal{E}_{2}$. Hence, $F_{1} \cap F_{2} \supset\left(\cap \mathcal{E}_{1}\right) \cap\left(\cap \mathcal{E}_{2}\right)$. We set $\mathcal{E}:=\mathcal{E}_{1} \cup \mathcal{E}_{2}$. Thus, $\mathcal{E} \subset\{B\} \cup \mathcal{S}$ is finite with $\left(\cap \mathcal{E}_{1}\right) \cap\left(\cap \mathcal{E}_{2}\right) \supset \cap \mathcal{E}$. By applying the hypothesis, we obtain $\mathcal{F} \in \cap\left\{N(F): F \in \mathcal{F} \cap \mathcal{B}^{X}\right\}$. Evidently, $\mathcal{S} \cap \mathcal{B}^{X} \subset \mathcal{F}$ is valid. $B \in \mathcal{F} \cap \mathcal{B}^{X}$ implies $\mathcal{F} \in N(B)$ and consequently, $\mathcal{S} \cap \mathcal{B}^{X} \in$ $N(B)$ follows. But then, $\mathcal{S} \in N(B)$ results, which contradicts.

Proposition 2.15. Let $(X, \xi)$ be a nearness space. Then, the following statements are equivalent:

(i) $(X, \xi)$ is totally bounded;

(ii) $\left(X, \underline{P} X, N_{\xi}\right)$ is full-bounded.

Proof. To (i) $\Longrightarrow$ (ii): Let $B \in \underline{P} X \backslash\{\emptyset\}$ and $\mathcal{S} \notin N_{\xi}(B)$. Hence, $\{B\} \cup \mathcal{S} \notin \xi$ and by the hypothesis, we can find $\mathcal{E} \subset\{B\} \cup \mathcal{S}$ finite with $\cap \mathcal{E}=\emptyset$. Thus, $B \cap(\cap \mathcal{E})=\emptyset$.

To (ii) $\Longrightarrow$ (i): Conversely, let $\left(\underline{P} X, N_{\xi}\right)$ be full-bounded. $\mathcal{S} \notin \xi$ implies $\mathcal{S} \neq \emptyset$. Choose $F \in \mathcal{S}$. Hence, $\{F\} \cup \mathcal{S} \notin N_{\xi}(X)$. By the hypothesis, we can find $\mathcal{E} \subset\{X\} \cup \mathcal{S}$ finite with $X \cap(\cap \mathcal{E})=\emptyset$ and consequently, $\cap \mathcal{E}=\emptyset$ follows.

In this context, we also infer that the property of being full-bounded is transferred by the completion process, too. Before proving this, we will give the following definition.

Definition 2.16. Let $\left(X, \mathcal{B}^{X}, N\right)$ be a pseudonear space, then $\mathcal{C} \subset \underline{P} X$ is called a unit (in $\left(\mathcal{B}^{X}, N\right)$ ), provided that $\mathcal{C}$ satisfies the following conditions: 
$\left(\right.$ ut $\left._{1}\right) \mathcal{C} \in \underline{P \mathcal{B}^{X}} \cap N(B)$ for some $B \in \mathcal{B}^{X} \backslash\{\emptyset\}$;

(ut ut $B \in \mathcal{C}$;

(ut ut $_{3} \supset B_{1} \supset \in \mathcal{C}, B_{1} \in \mathcal{B}^{X}$ implies $B_{1} \in \mathcal{C}$;

(ut u $_{4} B_{1}, B_{2} \notin \mathcal{C}, B_{1}, B_{2} \in \mathcal{B}^{X}$ implies $B_{1} \cup B_{2} \notin \mathcal{C}$;

(ut u $\operatorname{cl}_{N}(D) \in \mathcal{C}, D \in \mathcal{B}^{X}$ implies $D \in \mathcal{C}$.

Remark 2.17. First, we note that every $\mathrm{N}$-tape in $\mathcal{B}^{X}$ forms a unit in $\left(\mathcal{B}^{X}, N\right)$. And for a nearness $\xi$, the following statements are equivalent:

(i) $\mathcal{C} \subset \underline{P} X$ is a $\xi$-bunch;

(ii) $\mathcal{C} \subset \underline{P} X$ is a unit in $\left(\underline{P} X, N_{\xi}\right)$.

Lemma 2.18. For a pseudonear space $\left(X, \mathcal{B}^{X}, N\right)$, the below listed statements are equivalent:

(i) $\left(X, \mathcal{B}^{X}, N\right)$ is full-bounded;

(ii) $\left(X^{*}, \mathcal{B}^{X^{*}}, N^{*}\right)$ is full-bounded.

Proof. To (i) $\Longrightarrow$ (ii): Let $\mathcal{F}^{*} \in F I L\left(X^{*}\right) \backslash\left\{\underline{P} X^{*}\right\}$ with $F^{*} \in \mathcal{F}^{*} \cap \mathcal{B}^{X^{*}}$. Our goal is $\mathcal{F}^{*} \in N^{*}\left(F^{*}\right)$. We put $\mathcal{F}_{X}:=\left\{F \subset X: \exists A^{*} \in \mathcal{F}^{*} \cap \mathcal{B}^{X^{*}} F \supset j^{-1}\left[A^{*}\right]\right\}$. Note, for each $A^{*} \in \mathcal{F}^{*} \cap \mathcal{B}^{X^{*}}$ is $j^{-1}\left[A^{*}\right] \neq \emptyset$. In fact, let $A^{*} \in \mathcal{F}^{*} \cap \mathcal{B}^{X^{*}}$. Hence, $A^{*} \neq \emptyset$. Choose $\mathcal{C} \in A^{*}$, then by the hypothesis, we can find $x \in X$ with $\{x\} \in \mathcal{C}$. We will show $j(x)$ equals $\mathcal{C}$. $F \in j(x)$ implies $x \in c l_{N}(F)$. Hence, $c_{N}(F) \in \mathcal{C}$ follows, implying $F \in \mathcal{C}$ according to 2.17. Conversely, let $F \in \mathcal{C}$. But $\mathcal{C} \in \underline{P} \mathcal{B}^{X} \cap N(B)$ for some $B \in \mathcal{B}^{X} \backslash\{\emptyset\}$. Hence, by the symmetry of $\left(\mathcal{B}^{X}, N\right),\{B\} \cup \mathcal{C} \in N(F)$ follows. Thus, $\{F\} \cup(\{B\} \cup \mathcal{C}) \in N(\{x\})$ is valid, by applying again the symmetry of $\left(\mathcal{B}^{X}, N\right)$. Consequently, $\{F\} \in N(\{x\})$ implies $F \in j(x)$, showing the proposed equation. Since $j^{-1}\left[F^{*}\right] \in \mathcal{F}_{X}$, we have $\mathcal{F}_{X} \neq \emptyset$. Now, let $F_{1}, F_{2} \in \mathcal{F}_{X}$. Hence, there exists $A_{1}^{*}, A_{2}^{*} \in \mathcal{F}^{*} \cap \mathcal{B}^{X^{*}}$ with $F_{1} \supset j^{-1}\left[A_{1}^{*}\right]$ and $F_{2} \supset j^{-1}\left[A_{2}^{*}\right] . F_{1} \cap F_{2} \supset j^{-1}\left[A_{1}^{*}\right] \cap j^{-1}\left[A_{2}^{*}\right]=j^{-1}\left[A_{1}^{*} \cap A_{2}^{*}\right]$ with $A_{1}^{*} \cap A_{2}^{*} \in$ $\mathcal{F}^{*} \cap \mathcal{B}^{X^{*}}$. Hence, $F_{1} \cap F_{2} \in \mathcal{F}_{X}$ follows, showing that $\mathcal{F}_{X} \in F I L(X) \backslash\{\underline{P} X\}$. By the hypothesis, $\mathcal{F}_{X} \in N\left(j^{-1}\left[F^{*}\right]\right)$ is true, which implies $j \mathcal{F}_{X} \in N^{*}\left(j\left[j^{-1}\left[F^{*}\right]\right]\right) \subset$ $N^{*}\left(F^{*}\right)$ by applying Proposition 2.9. Now, we show $\mathcal{F}^{*} \cap \mathcal{B}^{X^{*}} \ll j \mathcal{F}_{X}$. Let $A^{*} \in \mathcal{F}^{*} \cap \mathcal{B}^{X^{*}}$, so $j^{-1}\left[A^{*}\right] \in \mathcal{F}_{X}$. Hence, $j\left[j^{-1}\left[A^{*}\right]\right] \in j \mathcal{F}$ with $A^{*} \supset j\left[j^{-1}\left[A^{*}\right]\right]$. Consequently, $\mathcal{F}^{*} \cap \mathcal{B}^{X^{*}} \in N^{*}\left(F^{*}\right)$ follows and $\mathcal{F}^{*} \in N^{*}\left(F^{*}\right)$ results because $\left(\mathcal{B}^{X^{*}}, N^{*}\right)$ is especially b-absorbed.

To (ii) $\Longrightarrow$ (i): For $\mathcal{F} \in F I L(X) \backslash\{\underline{P} X\}$, let $F \in \mathcal{F} \cap \mathcal{B}^{X}$. Our goal is $\mathcal{F} \in$ $N(F)$. We get $j[F] \in j(\mathcal{F}) \cap \mathcal{B}^{X^{*}}$ by applying Proposition 2.9. By the hypothesis, $j \mathcal{F} \in N^{*}(j[F])$ follows. Hence, there exists $B \in \mathcal{B}^{X} \backslash\{\emptyset\}$ such that $\mathcal{V}:=\left\{A \in \mathcal{B}^{X}\right.$ : $\left.\exists F^{*} \in\left(j \mathcal{F} \cap \mathcal{B}^{X^{*}}\right) \cup\{j[F]\}, A \in \Delta F^{*}\right\} \in N(B)$. Since $F \in \mathcal{V}$, note that $F \in \Delta j[F]$ is valid because $\mathcal{C} \in j[F]$ implies $\mathcal{C}=j(x)$ for some $x \in F$ and $x \in c l_{N}(F)$ is valid implying $F \in \mathcal{C}_{x}^{N}=j(x)=\mathcal{C}$. By the symmetry, of $\left(\mathcal{B}^{X}, N\right),\{B\} \cup \mathcal{V} \in N(F)$ is true. So it remains to verify that $\mathcal{F} \cap \mathcal{B}^{X} \subset \mathcal{V}$ can be deduced. But $A \in \mathcal{F} \cap \mathcal{B}^{X}$ implies $j[A] \in\left(j \mathcal{F} \cap B^{X^{*}}\right) \cup\{j[F]\}$ with $A \in \Delta j[A]$. Then, $\mathcal{F} \cap \mathcal{B}^{X} \in N(F)$ follows, implying $\mathcal{F} \in N(F)$ since $\left(\mathcal{B}^{X}, N\right)$ is especially b-absorbed, and the claim results. 


\section{The B-COMPACTIFICATION}

An important property in the theory of topological structures is that of being compact. Here, we especially note that the relatively compact subsets of a given topological space form a bornology. Now, if given a nearness space $(X, \xi)$ such that the underlying topology $\xi^{t}$ is Hausdorff, then we denote by $\mathcal{B}^{X}$ the set of all relatively $\xi^{t}$-compact subsets of $X$ and define an operator $N^{\xi^{t}}: \mathcal{B}^{X} \longrightarrow \underline{P}(\underline{P}(\underline{P} X))$ by setting:

$$
N^{\xi^{t}}(\emptyset):=\{\emptyset\}
$$

and

$$
N^{\xi^{t}}(B):=\left\{\mathcal{S} \subset \underline{P} X:\{B\} \cup\left(\mathcal{S} \cap \mathcal{B}^{X}\right) \in \xi^{t}\right\} \text { for every } B \in \mathcal{B}^{X} \backslash\{\emptyset\} .
$$

As a consequence, we get that the pair $\left(\mathcal{B}^{X}, N^{\xi^{t}}\right)$ forms a pseudonearness, which, in addition, is necessarily separated. Here, we will only verify the conditions for being hull-bounded and separated, respectively.

To (hb): Let $B \in \mathcal{B}^{X}$ with $x \in c l_{N \xi^{t}}(B)$. Hence, $\{B\} \in N^{\xi^{t}}(\{x\})$ is valid. By the definition of $N^{\xi^{t}}$, we obtain $\{\{x\}, B\}=\{\{x\}\} \cup\{B\} \in \xi^{t}$, which implies $x \in c l_{\xi^{t}}(B)$. By the hypothesis, $c l_{\xi^{t}}(B)$ is $\xi^{t}$-compact and we have $c l_{\xi^{t}}\left(c l_{N \xi^{t}}(B)\right) \subset$ $c l_{\xi^{t}}(B)$. Since $c l_{\xi^{t}}\left(c l_{N \xi^{t}}(B)\right)$ is a closed subset of $c l_{\xi^{t}}(B)$, it is $\xi^{t}$ compact because the underlying topology $\xi^{t}$ of $(X, \xi)$ is Hausdorff. Thus, $c l_{N^{t}}(B)$ is relatively compact and the claim follows.

To (sep): For elements $x, z \in X$, let $\{\{z\}\} \in N^{\xi^{t}}(\{x\})$. Hence, $\{\{x\},\{z\}\}=$ $\{\{x\}\} \cup\{\{z\}\} \in \xi^{t}$ follows. Note that $\xi^{t}:=\left\{\mathcal{A} \subset \underline{P} X: \cap\left\{c l_{\xi}: A \subset \mathcal{A}\right\} \neq \emptyset\right\}$ and, moreover, it is also a topological $N_{1}$-space. But then, by the hypothesis, $x=z$ results.

Definition 3.1. We call a pseudonearness $\left(\mathcal{B}^{X}, N\right)$ and the space $\left(X, \mathcal{B}^{X}, N\right)$ b-hullsected, provided they satisfy the following condition, i.e.

(b-hsc) $\forall \mathcal{S} \in \underline{P B}^{X}$ with $\cap\left\{c l_{N}(F): F \in \mathcal{S}\right\}=\emptyset, \exists \mathcal{S}_{0} \subset \mathcal{S}$ finite $\cap\left\{c l_{N}(A): A \in\right.$ $\left.\mathcal{S}_{0}\right\}=\emptyset$.

Remark 3.2. We note that every finite pseudonear space is b-hullsected.

Proposition 3.3. For a topoform pseudonear space $\left(X, \mathcal{B}^{X}, N\right)$, the following statements are equivalent:

(i) $\left(X, \mathcal{B}^{X}, N\right)$ is b-hullsected;

(ii) $\left(X, \mathcal{B}^{X}, N\right)$ is contiguous;

(iii) $\left(X, \mathcal{B}^{X}, N\right)$ is full-bounded.

Proof. To (i) $\Longrightarrow$ (ii): Let $B \in \mathcal{B}^{X} \backslash\{\emptyset\}$ and suppose $\mathcal{S} \notin N(B)$. Hence, $\cap\left\{c l_{N}(F): F \in\left(\mathcal{S} \cap \mathcal{B}^{X}\right) \cup\{B\}\right\}=\emptyset$. Otherwise, we can find $x \in c l_{N}(B)$ with $x \in \cap\left\{c l_{N}(F): F \in \mathcal{S} \cap \mathcal{B}^{X}\right\}$. Consequently, $\left\{c l_{N}(F): F \in \mathcal{S} \cap \mathcal{B}^{X}\right\} \in N(\{x\})$ with $N(\{x\}) \subset N\left(c l_{N}(B)\right)=N(B)$ implying $\mathcal{S} \cap \mathcal{B}^{X} \in N(B)$ and $\mathcal{S} \in N(B)$ follows, which contradicts. By the hypothesis, we can find $\mathcal{E} \subset(\{B\} \cup \mathcal{S}) \cap \mathcal{B}^{X}$ finite with $\cap\left\{c l_{N}(A): A \in \mathcal{E}\right\}=\emptyset$. But $\mathcal{E} \notin N(B)$ results because, otherwise, we obtain a contradiction by $\left(\mathcal{B}^{X}, N\right)$ being b-hullsected.

To (ii) $\Longrightarrow$ (iii): See Remark 2.14 . 
To (iii) $\Longrightarrow$ (i): If $\left(\mathcal{B}^{X}, N\right)$ is not b-hullsected, we can find $\mathcal{S} \in \underline{P \mathcal{B}^{X}}$ with $\cap\left\{c l_{N}(F): F \in \mathcal{S}\right\}=\emptyset$ and for each $\mathcal{S}_{0} \subset \mathcal{S}$ finite we have $\cap\left\{c l_{N}(A): A \in\right.$ $\left.\mathcal{S}_{0}\right\} \neq \emptyset$. We put $\mathcal{F}:=\left\{D \subset X: \exists \mathcal{E} \subset \mathcal{S}\right.$ finite $D \supset \cap\left\{c l_{N}(E): E \in \mathcal{E}\right\}$. Then, $\mathcal{F} \in F I L(X) \backslash\{\underline{P} X\}$ follows evidently. Now, if we choose $c l_{N}(F) \in \mathcal{F}$ for some $F \in \mathcal{S}$, we get $\mathcal{F} \in N\left(c l_{N}(F)\right)=N(F)$. Note that $\left(\mathcal{B}^{X}, N\right)$ is symmetric and full-bounded with $c l_{N}(F) \in \mathcal{B}^{X}$. Thus, we can find $x \in c l_{N}(F)$ such that $x \in \cap\left\{c l_{N}(D): D \in \mathcal{F} \cap \mathcal{B}^{X}\right\}$ since $\left(\mathcal{B}^{X}, N\right)$ is topoform. But for each $A \in \mathcal{S}$, $c l_{N}(A) \in \mathcal{F} \cap \mathcal{B}^{X}$ is valid. Hence, $x \in c l_{N}(A)$ follows, which contradicts.

Remark 3.4. By transforming this result to nearness spaces, we can now infer that for a topological nearness space $(X, \xi)$ the following properties are equivalent:

(i) $(X, \xi)$ is contigual;

(ii) $(X, \xi)$ is totally bounded;

(iii) $(X, \xi)$ is compact.

Proof. By applying the previous results.

Motivated by the just-obtained statements we are giving the following intrinsic definition:

Definition 3.5. We call a pseudonearness $\left(\mathcal{B}^{X}, N\right)$ and the space $\left(X, \mathcal{B}^{X}, N\right)$ $b$-compact, provided $\left(\mathcal{B}^{X}, N\right)$ is topoform and b-hullsected.

Remark 3.6. With respect to Remark 3.4, we note that for a nearness space $(X, \xi)$ the following statements are equivalent:

(i) $(X, \xi)$ is compact;

(ii) $\left(X, \underline{P} X, N_{\xi}\right)$ is b-compact.

In addition, with respect to Remark 3.2, we mention that every finite topoform pseudonear space is b-compact.

In this context, another important property comes into play.

Definition 3.7. A separated pseudonear space $\left(X, \mathcal{B}^{X}, N\right)$ is called precede, provided it satisfies the following condition:

(pc) $B \in \mathcal{B}^{X} \backslash\{\emptyset\}$ and $\mathcal{S} \in N(B)$ with $\mathcal{S} \cap \mathcal{B}^{X} \neq \emptyset$ implying the existence of an N-tape $\mathcal{T}$ in $\mathcal{B}^{X}$ such that $\mathcal{S} \cap \mathcal{B}^{X} \subset \mathcal{T}$.

Remark 3.8. Here, we point out that, in the case of saturation, precede pseudonear spaces and concrete $N_{1}$-spaces are essentially the same (cf. [1]).

Example 3.9. Every separated contiguous pseudonear space is precede.

Proof. For $B \in \mathcal{B}^{X} \backslash\{\emptyset\}$, let $\eta^{t} \subset N(B) \backslash\{\emptyset\}$ be a total ordered subset. We put $\cup\left\{\mathcal{A} \subset \underline{P} X: \mathcal{A} \in \eta^{t}\right\}=: \cup \eta^{t}$. Our goal is to verify $\cup \eta^{t} \in N(B) \backslash\{\emptyset\}$. If $\cup \eta^{t} \notin N(B)$, then we can find $\mathcal{E} \subset\{B\} \cup\left(\cup \eta^{t} \cap \mathcal{B}^{X}\right)$ finite with $\mathcal{E} \notin N(B)$ since, by the hypothesis, $\left(X, \mathcal{B}^{X}, N\right)$ is contiguous. Now, for each $E \in \mathcal{E}$, choose $\mathcal{A}_{E} \in \eta^{t} \cap \mathcal{B}$ with $E \in \mathcal{A}_{E}$ or $E=B$. We put $\xi:=\left\{\mathcal{A}_{E}: E \in \mathcal{E}\right\}$. Hence, $\xi \subset \eta^{t} \cap \mathcal{B}^{X}$ is finite and therefore, it possesses a smallest element $\mathcal{A}_{E}^{S}$. Consequently, $\mathcal{A}_{E}^{S} \in N(B) \backslash\{\emptyset\}$ is valid and, by the symmetry, we get $\{B\} \cup \mathcal{A}_{E}^{S} \in N(B) \backslash\{\emptyset\}$ with $\mathcal{E} \ll\{B\} \cup \mathcal{A}_{E}^{S}$, which contradicts. By using Zorn's lemma, we obtain that every non-empty $B$ pseudonear collection $\mathcal{S} \in N(B)$ is contained in a maximal element $\mathcal{C} \in N(B) \backslash\{\emptyset\}$. Consequently, $\emptyset \neq \mathcal{S} \cap \mathcal{B}^{X} \subset \mathcal{C} \cap \mathcal{B}^{X}=: \mathcal{T}$ follows, which shows the claim. 
Lemma 3.10. For a precede pseudonear space $\left(X, \mathcal{B}^{X}, M\right)$ its b-completion $\left(X^{*}, \mathcal{B}^{X^{*}}, M^{*}\right)$ is topoform.

Proof. For $B^{*} \in \mathcal{B}^{X^{*}} \backslash\{\emptyset\}$, let $\mathcal{A}^{*} \in M^{*}\left(B^{*}\right)$. Hence, there exists $B \in \mathcal{B}^{X} \backslash\{\emptyset\}$ such that $\mathcal{V}:=\left\{F \in \mathcal{B}^{X}: F \in \Delta A^{*}\right.$ for some $\left.A^{*} \in\left(\mathcal{A}^{*} \cap \mathcal{B}^{X^{*}}\right) \cup\left\{B^{*}\right\}\right\} \in M(B)$. By the symmetry, $\{B\} \cup \mathcal{V} \in M(B)$ implies $(\{B\} \cup \mathcal{V}) \cap \mathcal{B}^{X} \neq \emptyset$. Hence, by the hypothesis, we can find an N-tape $\mathcal{T}$ in $\mathcal{B}^{X}$ with $(\{B\} \cup \mathcal{V}) \cap \mathcal{B}^{X} \subset \mathcal{T}$. Since $\Delta B^{*} \cup \mathcal{T} \subset \mathcal{T}$ and $\Delta A^{*} \cup \mathcal{T} \subset \mathcal{T}$ for $A^{*} \in \mathcal{A}^{*} \cap \mathcal{B}^{X^{*}}$ are valid, we obtain the desired result.

Definition 3.11. (i) A pseudonear space $\left(X, \mathcal{B}^{X}, N\right)$ is called a pseudonear subspace of a pseudonear space $\left(Y, \mathcal{B}^{Y}, M\right)$, provided $X$ is a subset of $Y, \mathcal{B}^{X}$ is a subset of $\mathcal{B}^{Y}$ and for each $B \in \mathcal{B}^{X} \backslash\{\emptyset\}$ we have $\mathcal{S} \in N(B)$ iff $\mathcal{S} \in M(B)$ for every collection $\mathcal{S}$ of subsets of $X$.

(ii) Then, a separated pseudonear space $\left(Y, \mathcal{B}^{Y}, M\right)$ is called a $b$-compactification of a separated pseudonear space $\left(X, \mathcal{B}^{X}, N\right)$, provided that $\left(Y, \mathcal{B}^{Y}, M\right)$ is b-compact and $\left(X, \mathcal{B}^{X}, N\right)$ is a pseudonear subspace of $\left(Y, \mathcal{B}^{Y}, M\right)$ with $c l_{M}(X)=Y$.

(iii) A b-compactification $\left(Y, \mathcal{B}^{Y}, M\right)$ of a separated pseudonear space $\left(X, \mathcal{B}^{X}, N\right)$ is called strict, provided $\forall D \subset Y, D=c l_{M}(D)$ and $\forall y \notin D, \exists F \in \mathcal{B}^{X}$ such that $y \notin c l_{M}(F)$ and $D \subset c l_{M}(F)$.

Theorem 3.12. Every separated contiguous pseudonear space has a strict b-compactification.

Proof. Let $\left(X, \mathcal{B}^{X}, M\right)$ be a separated contiguous pseudonear space. Then, by Example 3.9 , it is precede. Thus, the b-completion $\left(X^{*}, \mathcal{B}^{X^{*}}, M^{*}\right)$ is topoform by applying Lemma 3.10. Furthermore, it is contiguous by using Proposition 2.12. But then, the b-completion is b-compact according to 3.3 with $\left(X, \mathcal{B}^{X}, M\right)$ being a pseudonear subspace of $\left(X^{*}, \mathcal{B}^{X^{*}}, M^{*}\right)$. Note that there is no need to distinguish, for a subset $A \subset X$, between $A$ and $j[A]$. It remains to prove that $\left(X^{*}, \mathcal{B}^{X^{*}}, M^{*}\right)$ is strict. Now, consider $A^{*} \subset X^{*}$ being closed with $\mathcal{T} \notin A^{*}$. Then, $\mathcal{T} \notin c l_{X^{*}}\left(A^{*}\right)$ implies $\left\{A^{*}\right\} \notin M^{*}(\{\mathcal{T}\})$. On the other hand, $\mathcal{T} \in \underline{P} \mathcal{B}^{X} \cap M(B) \backslash\{\emptyset\}$ for some $B \in \mathcal{B}^{X} \backslash\{\emptyset\}$ implies $\mathcal{V}:=\left\{A \in \mathcal{B}^{X}: \exists D^{*} \in\left(\left\{A^{*}\right\} \cap \mathcal{B}^{X^{*}}\right) \cup\{\{\mathcal{T}\}\}, A \in \Delta A^{*}\right\} \notin$ $M(B)$. Hence, $\Delta A^{*} \cup \mathcal{T} \nsubseteq \mathcal{T}$. Otherwise, since $\mathcal{V} \subset \Delta A^{*} \cup \mathcal{T}$ is valid, we get a contradiction. Consequently, we can find $F \in \Delta A^{*} \cup \mathcal{T}$ with $F \notin \mathcal{T}$. Hence, $F \in \Delta A^{*}$ follows. Our goal is to verify

(1) $\mathcal{T} \notin c l_{M^{*}}(j[F])$ and

(2) $A^{*} \subset \operatorname{cl}_{M^{*}}(j[F])$.

To (1): If $\mathcal{T} \in \operatorname{cl}_{M^{*}}(j[F])$, then $\{j[F]\} \in M^{*}(\{\mathcal{T})$ implies the existence of $D \in$ $\mathcal{B}^{X} \backslash\{\emptyset\}$ such that $m:=\left\{M \in \mathcal{B}^{X}: \exists D^{*} \in\{j[F]\} \cup\{\{\mathcal{T}\}\}, M \in \Delta D^{*}\right\} \in M(D)$. Hence, $\Delta j[F] \cup \mathcal{T} \subset m$ follows because $A \in \Delta j[F] \cup \mathcal{T}$ implies $A \in \Delta j[F]$ or $A \in \mathcal{T}=\Delta\{\mathcal{T}\}$. In both cases, $A \in m$ results. Thus, $\Delta j[F] \cup \mathcal{T} \in M(D)$ is valid. But $F \in \Delta j[F] \cup \mathcal{T}$ implies $\{F\} \cup \mathcal{T} \in M(D)$ and $F \in \mathcal{T}$ results since $\mathcal{T}$ satisfies $\left(\mathrm{tp}_{2}\right)$. But this contradicts.

To (2): For $\mathcal{D} \in A^{*}$, we have $F \in \mathcal{D}$. Hence, $\Delta j[F] \subset \mathcal{D}$. Note that $A \in \Delta j[F]$ implies $F \subset c l_{M}(A)$ and $c l_{M}(A) \in \mathcal{D}$ implies $A \in \mathcal{D}$. Observe that $\mathcal{D}$ is a unit in $\left(\mathcal{B}^{X}, M\right)$ according to 2.17 . Thus, $\mathcal{D} \in c_{M^{*}}(j[F])$. 
Remark 3.13. If we consider the saturated case, we can now infer that every separated contiguity space has a strict bicompact extension [4].

\section{The BORNOTOPOLOGICAL EXTENSION}

Closely related to the cononical construction which embeds each pseudonear space into a b-complete pseudonear space, we introduce the notion of a so-called bornotopological extension. It turns out that this concept is convenient for studying strict topological extensions. The main result is that we obtain a natural correspondence between equivalence classes of strict bornotopological extensions and precede pseudonear structures which is onto and one-to-one. In the case of separated contiguous pseudonear spaces, we can now infer that any strict $T_{1}$-compactification can be obtained in this way up to equivalence.

Definition 4.1. A bornotopological extension (in short a btop-extension) consists of a triple $\left(e, \mathcal{B}^{X}, Y\right)$, where $X:=\left(X, t_{X}\right), Y:=\left(Y, t_{Y}\right)$ are topological spaces (given by closure operators $t_{X}$ and $t_{Y}$, respectively), $\mathcal{B}^{X}$ is a bornology such that $B \in \mathcal{B}^{X}$ implies $t_{X}(B) \in \mathcal{B}^{X}$ and $e: X \longrightarrow Y$ is an injective map satisfying the following conditions:

$\left(\right.$ btx $\left._{1}\right) B \in \mathcal{B}^{X}$ implies $t_{X}(B)=e^{-1}\left[t_{Y}(e(B)]\right.$, where $e^{-1}$ denotes the inverse image under $e$;

$\left(\right.$ btx $\left._{2}\right) t_{Y}(e[X])=Y$, which means that the image of $X$ under $e$ is dense in $Y$.

Definition 4.2. In the above definition, a topological space means a $T_{1}$-space and all spaces in question are supposed to be not empty. Note also that if $\mathcal{B}^{X}$ is saturated, the above description and that of a topological extension in the usual sense coincide [1].

Lemma 4.3. For a bornotopological extension $\left(e, \mathcal{B}^{X}, Y\right),\left(\mathcal{B}^{X}, N_{e}\right)$ is a separated pseudonearness, where

$$
N_{e}(\emptyset):=\{\emptyset\}
$$

and

$$
N_{e}(B):=\left\{\mathcal{S} \subset \underline{P} X: \cap\left\{t_{Y}(e[F]): F \in\left(\mathcal{S} \cap \mathcal{B}^{X}\right) \cup\{B\} \neq \emptyset\right\} \text { if } B \in \mathcal{B}^{X} \backslash\{\emptyset\}\right.
$$

such that the triple $\left(X, \mathcal{B}^{X}, N_{e}\right)$ defines a separated pseudonear space with

$$
c l_{N_{e}}(B)=t_{X}(B) \quad \forall B \in \mathcal{B}^{X} .
$$

Definition 4.4. (i) btop-extensions $\left(e, \mathcal{B}^{X}, Y\right),\left(e^{\prime}, \mathcal{B}^{X}, Y^{\prime}\right)$ are called isovalent, provided that there exists a bijective map $h: Y \longrightarrow Y^{\prime}$ with $h \circ e=e^{\prime}$ such that $\forall D \in \mathcal{B}^{X} \forall y \in Y, y \in t_{Y}(e[D])$ iff $h(y) \in t_{Y}^{\prime}\left(e^{\prime}[D]\right)$;

(ii) equiform, provided that $N_{e}=N_{e^{\prime}}$ holds;

(iii) $\left(e, \mathcal{B}^{X}, Y\right)$ is called strict, provided $\forall D \subset Y, D=t_{Y}(D), \forall y \notin D \exists F \in \mathcal{B}^{X}$ such that $y \notin t_{Y}(e[F])$ and $D \subset t_{Y}(e[F])$ (compare with Definition 3.11);

(iv) for a pseudonear space $\left(X, \mathcal{B}^{X}, M\right)$ we say that the pseudonearness $\left(\mathcal{B}^{X}, M\right)$ is induced by a btop-extension, provided that there exists a btop-extension $\left(e, \mathcal{B}^{X}, Y\right)$ such that $M=N_{e}$.

Remark 4.5. Here, we note that if $\mathcal{B}^{X}$ is saturated, strict topological extensions and strict btop-extensions are essentially the same. Furthermore, we note 
that any separated topoform pseudonearness $\left(\mathcal{B}^{X}, M\right)$ is induced by $\left(i d_{X}, \mathcal{B}^{X}, X\right)$ with $i d_{X}: X \longrightarrow X$ denoting the identity and $X:=\left(X, c l_{M}\right)$.

Furthermore, for a separated topoform pseudonearness $\left(\mathcal{B}^{X}, M\right)$, we conclude that the btop-extensions $\left(i d_{X}, \mathcal{B}^{X}, X\right)$ and $\left(j, \mathcal{B}^{X}, X^{*}\right)$ are isovalent by applying 2.6 and 2.10. And finally, we infer that isovalent btop-extensions are equiform. In fact, let $\left(e, \mathcal{B}^{X}, Y\right),\left(e^{\prime}, \mathcal{B}^{X}, Y^{\prime}\right)$ be isovalent btop-extensions. We denote by $h: Y \longrightarrow Y^{\prime}$ the existing bijective map with its corresponding property. For $B \in \mathcal{B}^{X} \backslash\{\emptyset\}$, let $\mathcal{S} \in N_{e}(B)$. Then, by the definition of $N_{e}, \cap\left\{t_{Y}(e[F]): F \in\right.$ $\left.\left(\mathcal{S} \cap \mathcal{B}^{X}\right) \cup\{B\}\right\} \neq \emptyset$. Choose $y \in Y$ such that for $A \in\left(\mathcal{S} \cap \mathcal{B}^{X}\right) \cup\{B\}, y \in t_{Y}(e[A])$. Hence, $h(y) \in t_{Y^{\prime}}\left(e^{\prime}[A]\right)$ follows by applying the hypothesis. Consequently, $\mathcal{S} \in$ $N_{e^{\prime}}(B)$ results immediately. Vice versa, we use the inverse function of $h$.

Lemma 4.6. Let $\left(X, \mathcal{B}^{X}, M\right)$ be a pseudonear space induced by a strict btopextension. Then, $\left(X, \mathcal{B}^{X}, M\right)$ is precede (compare with Definition 3.7).

Proof. See [6].

Proposition 4.7. For a pseudonear space $\left(X, \mathcal{B}^{X}, M\right)$, let us denote by

$$
\left(X^{*}, \mathcal{B}^{X^{*}}, M^{*}\right)
$$

its corresponding b-completion. Then, for every $\mathcal{T} \in X^{*}$ and $D \in \mathcal{B}^{X}$ the following statements are equivalent:

(i) $\mathcal{T} \in c l_{M^{*}}(j[D])$;

(ii) $D \in \mathcal{T}$.

Proof. See [6].

Lemma 4.8. For a pseudonear space $\left(X, \mathcal{B}^{X}, M\right)$, let its b-completion

$$
\left(X^{*}, \mathcal{B}^{X^{*}}, M^{*}\right)
$$

be topoform. Then, $\left(j, \mathcal{B}^{X}, X^{*}\right)=: E$ is a strict btop-extension such that

$$
\left(X, \mathcal{B}^{X}, M\right)
$$

is induced by $E$.

Proof. Here, $E$ consists of $X:=\left(X, c l_{M}\right), X^{*}:=\left(X^{*}, c l_{M^{*}}\right)$ and $j: X \longrightarrow X^{*}$ as the canonical embedding. For the strictness condition see proof of Theorem 3.12. Evidently, $E$ satisfies the conditions $\left(b x_{1}\right)$ and $\left(b x_{2}\right)$ in Definition 4.1 . Thus, we have to verify that $M$ equals $N_{j}$ (see Lemma 4.3).

To " $M \leq N_{j}$ ": For $B \in \mathcal{B}^{X} \backslash\{\emptyset\}$, let $\mathcal{S} \in M(B)$. Hence, by Proposition 2.9, $j \mathcal{S} \in M^{*}(j[B])$. By the hypothesis, we can find $\mathcal{T} \in c l_{M^{*}}(j[B]), \mathcal{T} \in \cap\left\{c l_{M^{*}}(A)\right.$ : $\left.A \in j \mathcal{S} \cap \mathcal{B}^{X^{*}}\right\}$. Now, let $F \in \mathcal{S} \cap \mathcal{B}^{X}$. Then, $j[F] \in j \mathcal{S} \cap \mathcal{B}^{X^{*}}$ follows and $\mathcal{T} \in \operatorname{cl}_{M^{*}}(j[F])$ results. On the other hand, $\mathcal{T} \in \operatorname{cl}_{M^{*}}(j[B])$ closes this part of the proof.

To " $N_{j} \leq M$ ": Conversely, let $\mathcal{S} \in N_{j}(B)$. Hence, we can find $\mathcal{T} \in c l_{M^{*}}(j[B])$ with $\mathcal{T} \in \cap\left\{c l_{M^{*}}(j[F]): F \in \mathcal{S} \cap \mathcal{B}^{X}\right\}$. By applying Proposition 4.7., $B \in \mathcal{T}$ and $\mathcal{T} \in N(D)$ are valid for some $D \in \mathcal{B}^{X} \backslash\{\emptyset\}$. By the symmetry, $\{D\} \cup \mathcal{T} \in M(B)$ implies $\mathcal{T} \in M(B)$. But $F \in \mathcal{S} \cap \mathcal{B}^{X}$ implies $\mathcal{T} \in c_{M^{*}}(j[F])$ and, by Proposition 4.7, $F \in \mathcal{T}$ results, showing that $\mathcal{S} \cap \mathcal{B}^{X} \in M(B)$ is valid. Consequently, $\mathcal{S} \in M(B)$ $\operatorname{since}\left(\mathcal{B}^{X}, M\right)$ is b-absorbed. 
Theorem 4.9. For any pseudonear space $\left(X, \mathcal{B}^{X}, M\right)$ the following conditions are equivalent:

(i) $\left(\mathcal{B}^{X}, M\right)$ is a pseudonearness induced by a strict btop-extension;

(ii) The b-completion $\left(X^{*}, \mathcal{B}^{X^{*}}, M^{*}\right)$ of $\left(X, \mathcal{B}^{X}, M\right)$ is topoform;

(iii) $\left(X, \mathcal{B}^{X}, M\right)$ is a precede pseudonear space.

Proof. By applying the previous results in Lemmas 4.6, 3.10, and 4.8, respectively.

Corollary 4.10. If $\left(\mathcal{B}^{X}, M\right)$ is the pseudonearness induced by a strict btopextension $\left(e, \mathcal{B}^{X}, Y\right)$, then $\left(e, \mathcal{B}^{X}, Y\right)$ and $\left(j, \mathcal{B}^{X}, X^{*}\right)$ are equiform.

Proof. By the hypothesis, we get $N_{e}=M=N_{j}$.

Proposition 4.11. Let strict btop-extensions

$$
\left(e, \mathcal{B}^{X}, Y\right), \quad\left(e^{\prime}, \mathcal{B}^{X}, Y^{\prime}\right)
$$

be equiform such that $\mathcal{B}^{X}$ is saturated. Then, $\left(e, \mathcal{B}^{X}, Y\right),\left(e^{\prime}, \mathcal{B}^{X}, Y^{\prime}\right)$ are isovalent.

Proof. By the hypothesis, $N_{e}=N_{e^{\prime}}$. Hence, $N_{e}=N_{j}$ with $\left(j, \mathcal{B}^{X}, X^{*}\right)$, where $\left(X^{*}, \mathcal{B}^{X^{*}}, X^{*}\right)$ denotes the b-completion of $\left(X, \mathcal{B}^{X}, N_{e}\right)$. We define a map $h$ : $Y \longrightarrow X^{*}$ by setting for each $y \in Y: h(y):=\mathcal{T}^{y}:=\left\{D \in \mathcal{B}^{X}: y \in t_{Y}(e[D])\right\}$. $\mathcal{T}^{y}$ is an $N_{e}$-tape in $\mathcal{B}^{X}$ since $\mathcal{T} \in \underline{P} X \cap N_{e}(X) \backslash\{\emptyset\}$ is valid and $\{y\} \in \mathcal{T}^{y}$ by applying strictness. Further, note that $\mathcal{B}^{X}$ is saturated. Now, let $\{A\} \cup \mathcal{T}^{y} \in N(B)$ for some $B \in \mathcal{B}^{X} \backslash\{\emptyset\}$ and $A \in \mathcal{B}^{X}$. Then, $\{A,\{y\}\} \cup \mathcal{T}^{y} \in N_{e}(B)$ holds, and by applying the symmetry, we get $\{B\} \cup\left(\left\{A,\{\{y\}\} \cup \mathcal{T}^{y}\right) \in N_{e}(\{y\})\right.$. Hence, $\{A\} \in N_{e}(\{y\})$ implies $y \in t_{Y}\left(e[A)\right.$ and thus, $A \in \mathcal{T}^{y}$. Moreover, $h$ is bijective and satisfies the condition in Definition 4.4 (i), see also [6]. Thus, $h: Y \longrightarrow X^{*}$ is a homeomorphism and $Y \widetilde{h} X^{*}$ results.

Analogously, we obtain $Y^{\prime} \widetilde{h} X^{*}$. Hence, $Y \widetilde{h} Y^{\prime}$ is valid and the claim results.

Corollary 4.12. For strict btop-extensions $\left(e, \mathcal{B}^{X}, Y\right),\left(e^{\prime}, \mathcal{B}^{X}, Y^{\prime}\right)$ such that $\mathcal{B}^{X}$ is saturated, the following statements are equivalent:

(i) There exists a homeomorphism $h: Y \longrightarrow Y^{\prime}$ with $h \circ e=e^{\prime}$;

(ii) $\left(e, \mathcal{B}^{X}, Y\right),\left(e^{\prime}, \mathcal{B}^{X}, Y^{\prime}\right)$ are equiform.

Proof: By applying Remark 4.5, Corollary 4.10, and Propsition 4.11, respectively.

Remark 4.13. The above description rectifies and renews some corresponding statements in [6].

Remark 4.14. By applying Theorem 3.12 and Corollary 4.10, respectively, we can now state that every separated contiguous pseudonear space has a strict bcompactification. Vice versa, each strict btop-extension inducing such a space implies that this one and that of its strict b-compactification are equiform. That immediately implies a natural bijection between all separated contiguous pseudonear spaces and all equivalence classes of strict b-compactifications which are equiform to each other. Further, we point out that, in the saturated case, a corresponding result has already been published by Bentley and Herrlich, [1]. But according to 
Remark 3.13, our result also represents a generalization of a corresponding theorem for contiguity spaces and bicompact extensions in [4].

In addition, take into account that, in this case, a strict b-compactification consists of a strict btop-extension $\left(e, \mathcal{B}^{X}, Y\right)$ such that $Y$ is a compact topological space (see Remark 4.5).

In [8], the authors consider strongly far proximity based on a Lodato proximity. They continue with so-called hit and far-miss topologies related to the above topology on the hyperspace of non-empty closed subsets of a set $X$. Then, compactness comes into play by considering hypertopologies which are not comparable. Now, in the following, we look at the corresponding proxiform pseudonearness. First, we note the following proposition:

Proposition 4.15. For a btop-extension $\left(e, \mathcal{B}^{X}, Y\right)$ we set for $B_{1}, B_{2} \in \mathcal{B}^{X}$, $B_{1} \gamma_{e} B_{2}$ iff $t_{Y}\left(e\left[B_{1}\right]\right) \cap t_{Y}\left(e\left[B_{2}\right]\right) \neq \emptyset$. Then, $\left(X, \mathcal{B}^{X}, \gamma_{e}\right)$ forms a separated pseudoproximity space.

Proof. Here, we will only show the axiom $\left(\operatorname{psp}_{7}\right)$ of a pseudoproximity.

For $B, D \in \mathcal{B}^{X}$, let $B \gamma_{e} c l_{\gamma_{e}}(D)$. Hence, $t_{Y}(e[B]) \cap t_{Y}\left(e\left[c l_{\gamma_{e}}(D)\right]\right) \neq \emptyset$. But $c l_{\gamma_{e}}[D] \subset t^{X}(D)$ because $x \in \operatorname{cl}_{\gamma_{e}}(D)$ implies $\{x\} \gamma_{e} D$ and consequently,

$$
t_{Y}(\{e(x)\}) \cap t_{Y}(e[D]) \neq \emptyset \text {. }
$$

So, we can find $y \in t_{Y}(e[D])$ with $y \in t_{Y}(\{e(x)\}) . y=e(x)$ follows since $t_{Y}$ satisfies $\mathrm{T}_{1}$ and $e(x) \in t_{Y}(e[D])$ implies $x \in e^{-1}\left[t_{Y}(e[D])\right]=t_{X}(D)$. Hence, $e\left[c l_{\gamma_{e}}(D)\right] \subset$ $e\left[t_{X}(D)\right] \subset t_{Y}(e[D])$ follows and, by $\left(\operatorname{btex}_{1}\right), t_{Y}\left(e\left[c l_{\gamma_{e}}(D)\right]\right) \subset t_{Y}(e[D])$ is valid because $t_{Y}$ is topological. Finally, $t_{Y}(e[B]) \cap t_{Y}(e[D]) \neq \emptyset$ results, showing that $B \gamma_{e} D$ is valid.

As already shown, there exist a great deal of structures induced by some btopextensions. In the following section, we will characterize pseudonear spaces.

Definition 4.16. For a pseudonear space $\left(X, \mathcal{B}^{X}, M\right), \underline{G} \subset \underline{P} X$ is called an $M$-grill in $\mathcal{B}^{X}$, provided it satisfies the following conditions:

$\left(\operatorname{grl}_{1}\right) \underline{G} \in \underline{P} \mathcal{B}^{X} \cap M(B)$ for some $B \in \mathcal{B}^{X} \backslash\{\emptyset\}$;

$\left(\operatorname{grl}_{2}\right) B \in \underline{G}$

$\left(\mathrm{grl}_{3}\right) B_{1} \cup B_{2} \in \underline{G}$ iff $B_{1}, B_{2} \in \underline{G}$.

Remark 4.17. Note that each unit in $\left(\mathcal{B}^{X}, M\right)$ is an $M$-grill in $\mathcal{B}^{X}$ and so is every N-tape. Moreover, in the saturated case, for $\underline{G} \subset \underline{P} X$, the following statements are equivalent:

(i) $\underline{\mathrm{G}}$ is an $M$-grill in $\mathcal{B}^{X}$;

(ii) $\underline{\mathrm{G}}$ is an $\eta_{M}$-grill [1].

Definition 4.18. A pseudonear space $\left(X, \mathcal{B}^{X}, M\right)$ is called grillformic, provided it satisfies the following condition:

(grlf) $B \in \mathcal{B}^{X} \backslash\{\emptyset\}$ and $\mathcal{S} \in M(B)$ with $\mathcal{S} \cap \mathcal{B}^{X} \neq \emptyset$ imply the existence of an $M$-grill $\underline{\mathrm{G}}$ in $\mathcal{B}^{X}$ such that $\mathcal{S} \cap \mathcal{B}^{X} \subset \underline{G}$.

Remark 4.19. Especially note that each precede pseudonear space is grillformic. 
Theorem 4.20. For any pseudonear space $\left(X, \mathcal{B}^{X}, M\right)$ the following conditions are equivalent:

(i) $\left(X, \mathcal{B}^{X}, M\right)$ is induced by a btop-extension;

(ii) $\left(X, \mathcal{B}^{X}, M\right)$ is grillformic;

(iii) $\left(X, \mathcal{B}^{X}, M\right)$ is a pseudonear subspace of some topoform pseudonear space.

Proof. To (i) $\Longrightarrow$ (ii): By the hypothesis, there exists a btop-extension

$$
\left(e, \mathcal{B}^{X}, Y\right)
$$

with $M=N_{e}$. For $\mathcal{S} \in M(B)$ with $\mathcal{S} \cap \mathcal{B}^{X} \neq \emptyset$, we have $\cap\left\{t_{Y}(e[F]): F \in\right.$ $\left.\left(\mathcal{S} \cap \mathcal{B}^{X}\right) \cup\{B\}\right\} \neq \emptyset$. Consequently, we can find $y \in t_{Y}(e[B])$ such that $y \in$ $\cap\left\{t_{Y}(e[F]): F \in\left(\mathcal{S} \cap \mathcal{B}^{X}\right)\right.$. We put $t(y):=\left\{F \in \mathcal{B}^{X}: y \in t_{Y}(e[F])\right\}$. But

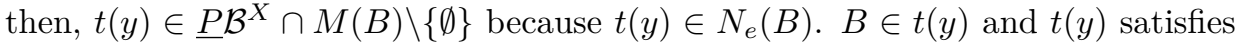
$\left(\operatorname{grl}_{3}\right)$ implying $t(y)$ is an $M$-grill in $\mathcal{B}^{X}$. Now, for $F \in \mathcal{S} \cap \mathcal{B}^{X}$, we have $F \in$ $\left(\mathcal{S} \cap \mathcal{B}^{X}\right) \cup\{B\}$. Hence, $y \in t_{Y}(e[F])$ implies $\mathcal{S} \cap \mathcal{B}^{X} \subset t(y)$.

To (ii) $\Longrightarrow$ (i): For any $M$ grill $\underline{G}$ in $\mathcal{B}^{X}, \mathcal{C}:=\left\{F \in \mathcal{B}: c l_{M}(F) \in \underline{G}\right\}$ defines an $M$-grill in $\mathcal{B}^{X}$ with $\underline{G} \subset \mathcal{C}$ such that

(E) $F \in \mathcal{C}$ iff $c l_{M}(F) \in \mathcal{C}$.

Let $\mathcal{G}:=\left\{\mathcal{C}: \mathcal{C}\right.$ is $M$-grill in $\mathcal{B}^{X}$ with $(\mathrm{E})$ and $\left.\cap\left\{c l_{M}(F): F \in \mathcal{C}\right\}=\emptyset\right\}$. We set $Y:=X \dot{\cup} \mathcal{G}$ as a disjoint union. Then, we define a topological closure operator $c l_{Y}$ on $Y$ by setting:

$$
\begin{aligned}
& y \in \operatorname{cl}_{Y}(A) \quad \text { iff } \quad y \in \operatorname{cl}_{M}(A \cap X) \quad \text { if } \quad y \in X \\
& y \in \operatorname{cl}_{Y}(A) \quad \text { iff } \quad y \in A \text { or } A \cap X \in \mathcal{G} \quad \text { if } \quad y \in \mathcal{G} \text {. }
\end{aligned}
$$

By regarding $e: X \longrightarrow Y$ as the inclusion, $\left(\mathcal{B}^{X}, M\right)$ is a pseudonearness induced by the extension $\left(e, \mathcal{B}^{X}, Y\right)$ with $X:=\left(X, c l_{M}\right)$ and $Y:=\left(Y, c l_{Y}\right)$.

To $(i) \Longrightarrow($ iii $)$ : Let $\left(e, \mathcal{B}^{X}, Y\right)$ be a btop-extension inducing $\left(X, \mathcal{B}^{X}, M\right)$. Hence, $M=N_{e}$ is valid. We regard $\left(\underline{P Y}, N t_{Y}\right)$ as defined in 1.18. Then, $\left(Y, \underline{P} Y, N_{t_{Y}}\right)$ is a topoform pseudonear space which contains $(X, \mathcal{B}, M)$ as proposed above.

To (iii) $\Longrightarrow$ (ii): Now, let the condition in (iii) be given such that $\left(X, \mathcal{B}^{X}, M\right)$ is a pseudonear subspace of some topoform pseudonear space $\left(Y, \mathcal{B}^{X}, N\right)$. $\mathcal{S} \in M(B)$, $B \in \mathcal{B}^{X} \backslash\{\emptyset\}$ with $\mathcal{S} \cap \mathcal{B}^{X} \neq \emptyset$ and $\mathcal{S} \in N(B)$ implying $\cap\left\{c l_{N}(F): F \in\left(\mathcal{S} \cap \mathcal{B}^{Y}\right) \cup\right.$ $\{B\}\} \neq \emptyset$. Choose $y \in c l_{N}(B)$ such that $y \in \cap\left\{c l_{N}(F): F \in \mathcal{S} \cap \mathcal{B}^{Y}\right\}$ and put $t(y):=\left\{A \in \mathcal{B}^{Y}: y \in c l_{N}(A)\right\}$. Hence, $t(y) \ll\{\{y\}\}$ with $\{\{y\}\} \in N(\{y\}) \subset$ $N\left(c_{N}(B)\right)=N(B)$. Consequently, $t(y) \cap \mathcal{B}^{X}=: \mathcal{T} \in M(B) \backslash\{\emptyset\}$ with $B \in \mathcal{T}$ such that $\mathcal{S} \cap \mathcal{B}^{X} \subset \mathcal{T}$. Evidently, $\mathcal{T}$ satisfies $\left(\operatorname{grl}_{3}\right)$ and the claim follows.

Now, returning to our previous concept, we can finally state that every separated proxiform pseudonear space has a strict b-compactification which, in addition, is separated. Moreover, in the case of saturation, that can now be interpreted as a comparative form of Lodato's famous theorem in [7].

On the other hand, there exist b-compactifications $\left(e, \mathcal{B}^{X}, Y\right)$ whose induced pseudonearness on $X$ is not proxiform, see [1]. Note that here, the spaces in question are being saturated. But now, at the end, we still offer the following two propositions. 
Proposition 4.21. For a topoform pseudonear space $\left(Y, \mathcal{B}^{Y}, N\right)$ the following statements are equivalent:

(i) $\left(Y, \mathcal{B}^{Y}, N\right)$ is proxiform;

(ii) $B \in \mathcal{B}^{Y} \backslash\{\emptyset\}$ and $\mathcal{S} \subset \underline{P} Y$ such that $\forall \mathcal{E} \subset\left(\mathcal{S} \cap \mathcal{B}^{Y}\right) \cup\{B\}$ finite $\exists A, D \in \mathcal{B}^{Y}$ with $c l_{N}(A) \cap c l_{N}(D) \neq \emptyset$ and $\left\{c l_{N}(E): E \in \mathcal{E}\right\} \ll\{A, D\}$, then $\mathcal{S} \in N(B)$.

Proof. To (ii) $\Longrightarrow$ (i): Let $B \in \mathcal{B}^{Y} \backslash\{\emptyset\}$ and $\mathcal{S} \subset \underline{P} Y$ such that for each $\mathcal{E} \subset\left(\mathcal{S} \cap \mathcal{B}^{Y}\right) \cup\{B\}$ finite there exist $A, D \in \mathcal{B}^{Y}$ with $A \gamma_{N} D$ and $\left\{c l_{N}(E): E \in\right.$ $\mathcal{E}\} \ll\{A, D\}$. Our goal is $\mathcal{S} \in N(B)$. By the hypothesis, $\{A, D\} \in N(A)$ implies $c l_{N}(A) \cap c l_{N}(D) \neq \emptyset$ since $\left(\mathcal{B}^{Y}, N\right)$ is topoform.

To (i) $\Longrightarrow$ (ii): Let $B \in \mathcal{B}^{Y} \backslash\{\emptyset\}$ and $\mathcal{S} \subset \underline{P Y}$ such that $\forall \mathcal{E} \subset\left(\mathcal{S} \cap \mathcal{B}^{Y}\right) \cup$ $\{B\}$ finite $\forall A, D \in \mathcal{B}^{Y}$ with $c l_{N}(A) \cap c l_{N}(D) \neq \emptyset$. Our goal is $\mathcal{S} \in N(B)$. By the hypothesis, we can find $y \in c l_{N}(A)$ with $y \in c l_{N}(D)$. Consequently, $\left\{c l_{N}(A), c l_{N}(D)\right\} \ll\{\{y\}\} \in N(\{y\}) \subset N\left(c l_{N}(A)\right)=N(A) \cap N(D)=N\left(c l_{N}(D)\right)$ and $\{A, D\} \in N(A) \cap N(D)$ results, which shows that $A \gamma_{N} D$ is valid.

Proposition 4.22. Let $\left(Y, \mathcal{B}^{Y}, N\right)$ be a proxiform b-compactification of a pseudonear space $\left(X, \mathcal{B}^{X}, M\right)$. Then, $\left(\mathcal{B}^{X}, M\right)$ is proxiform.

Proof. Let $\mathcal{S} \subset \underline{P} X$ and $B \in \mathcal{B}^{X} \backslash\{\emptyset\}$ such that for each $\mathcal{E} \subset\left(\mathcal{S} \cap \mathcal{B}^{X}\right) \cup\{B\}$ finite there exist $A, D \in \mathcal{B}^{X}$ with $A \gamma_{M} D$ and $\left\{c l_{M}(E): E \in \mathcal{E}\right\} \ll\{A, D\}$. Our goal is $\mathcal{S} \in M(B)$. If $\mathcal{S} \notin M(B), j \mathcal{S} \notin N(j[B])$, where $j: X \longrightarrow Y$ denotes the corresponding embedding, which is an injective bin-map satisfying $\mathcal{S} \in M(B)$ iff $j \mathcal{S} \in N(j[B]) \forall B \in \mathcal{B}^{X} \backslash\{\emptyset\}$. Then, by the hypothesis, we can find $\mathcal{E} \subset\left(j \mathcal{S} \cap \mathcal{B}^{Y}\right) \cup j[B]$ finite such that $\forall A, D \in \mathcal{B}^{Y}$ with $A \gamma_{N} D$ we have $\left\{c l_{N}(E): E \in \mathcal{E}\right\}<\nless\{A, D\}$. Hence, $c_{N}(E) \nsupseteq A, D$ for some $E \in j \mathcal{S} \cap \mathcal{B}^{Y}$. By setting $\mathcal{E}_{1}:=\left\{j^{-1}[E]\right\}, \mathcal{E}_{1} \subset\left(\mathcal{S} \cap \mathcal{B}^{X}\right) \cup\{B\}$ is finite. Consequently, $A_{1} \gamma_{M} D_{1}$ for some $A_{1}, D_{1} \in \mathcal{B}^{X}$ with $j^{-1}[E] \supset A_{1}$ or $j^{-1}[E] \supset D_{1}$. Hence, $j\left[A_{1}\right] \gamma_{N} j\left[D_{1}\right]$ with $E \supset j\left[A_{1}\right]$ or $E \supset j\left[D_{1}\right]$ implying $c l_{N}(E) \supset j\left[A_{1}\right]$ or $c l_{N}(E) \supset j\left[D_{1}\right]$, which contradicts and $\mathcal{S} \in M(B)$ follows, which shows that $\left(\mathcal{B}^{X}, M\right)$ is proxiform.

Last statement. Let $\left(X, \mathcal{B}^{X}, M\right)$ be a separated proxiform pseudonear space such that $\left(\mathcal{B}^{X}, M\right)$ is, in addition, topoform. Then, $\left(X, \mathcal{B}^{X}, M\right)$ is induced by a strict b-compactification which, additionally, is proxiform.

Proof. Let us consider $E:=\left(j, \mathcal{B}^{X}, X^{*}\right)$, then by Lemmas $1.30,3.10$, Theorem 3.12 , and Lemma 4.8 , respectively, $\left(X^{*}, \mathcal{B}^{X^{*}}, M^{*}\right)$ is a strict b-compactification such that $\left(X, \mathcal{B}^{X}, M\right)$ is induced by $E$. And since $j: X \longrightarrow X^{*}$ is bijective with $\left(\mathcal{B}^{X}, M\right)$ being proxiform, $\left(\mathcal{B}^{X^{*}}, M^{*}\right)$ is proxiform, too.

Note that for pseudonear spaces $\left(X, \mathcal{B}^{X}, M\right), \quad\left(Y, \mathcal{B}^{Y}, N\right)$ such that $\left(X, \mathcal{B}^{X}, M\right)$ is a pseudonear subspace of $\left(Y, \mathcal{B}^{Y}, N\right)$ with $\left(\mathcal{B}^{X}, M\right)$ being proxiform and $j: X \longrightarrow Y$ a bijective bin-map satisfying $\mathcal{S} \in M(B)$ iff $j \mathcal{S} \in N(j[B])$ for each $B \in \mathcal{B}^{X} \backslash\{\emptyset\},\left(\mathcal{B}^{X}, N\right)$ is proxiform, too.

\section{REFERENCES}

[1] H. L. Bentley and H. Herrlich, Extensions of topological spaces, Topology, Proceedings of the Memphis State University Conference 1975, 1976, pp. 129-184.

[2] H. Herrlich, A concept of nearness, General Topology and its Applications 4 (1974), 191212. 
[3] H. Hogbe-Nlend, Bornologies and Functional Analysis, North-Holland, Amsterdam, 1977.

[4] V.M. Ivanova and A. A. Ivanov, Contiguity spaces and bicompact extensions, Doklady Akademii Nauk SSSR 127 (1959), 20-22.

[5] C. Kuratowski, Sur l'opération $\bar{A}$ de l'analysis situs, Fundamenta Mathematicae 3 (1922), 182-199.

[6] D. Leseberg and Z. Vaziry, Bounded Topology, LAP Lambert Academic Publishing, 2019.

[7] M. W. Lodato, On topological induced generalized proximity relations II, Pacific journal of Mathematics 17 (1966), 131-135.

[8] J.F. Peters and C. Guadagni, Strongly far proximity and hyperspace topology, arXiv:1502.02771 (2015), 5 pp.

Dieter Leseberg, Ernst Reuter Gesellschaft Berlin, Germany e-mail: leseberg@zedat.fu-berlin.de

Zohreh Vaziry, Department of Mathematics, Karaj Branch, Islamic Azad University, Karaj, Iran

e-mail: z_m_vaziry@yahoo.co.in 
